\title{
Testing Yukawa-unified SUSY during year 1 of LHC: the role of multiple $b$-jets, dileptons and missing $E_{T}$
}

\author{
Howard Baer, ${ }^{a}$ Sabine Kraml, ${ }^{b}$ Andre Lessa ${ }^{a}$ and Sezen Sekmen ${ }^{c}$ \\ ${ }^{a}$ Dept. of Physics and Astronomy, University of Oklahoma, \\ Norman, OK 73019, U.S.A. \\ ${ }^{b}$ Laboratoire de Physique Subatomique et de Cosmologie, UJF Grenoble 1, \\ CNRS/IN2P3, INPG, 53 Avenue des Martyrs, F-38026 Grenoble, France \\ ${ }^{c}$ Dept. of Physics, Florida State University, \\ Tallahassee, FL 32306, U.S.A. \\ E-mail: baer@nhn.ou.edu, sabine.kraml@lpsc.in2p3.fr, lessa@nhn.ou.edu, \\ sezen.sekmen@cern.ch
}

Abstract: We examine the prospects for testing SO(10) Yukawa-unified supersymmetric models during the first year of LHC running at $\sqrt{s}=7 \mathrm{TeV}$, assuming integrated luminosity values of $\sim 0.1-1 \mathrm{fb}^{-1}$. We consider two cases: the Higgs splitting (HS) and the $D$-term splitting (DR3) models. Each generically predicts light gluinos and heavy squarks, with an inverted scalar mass hierarchy. We hence expect large rates for gluino pair production followed by decays to final states with large $b$-jet multiplicity. For $0.2 \mathrm{fb}^{-1}$ of integrated luminosity, we find a $5 \sigma$ discovery reach of $m_{\tilde{g}} \sim 400 \mathrm{GeV}$ even if missing transverse energy, $E_{T}^{\text {miss }}$, is not a viable cut variable, by examining the multi-b-jet final state. A corroborating signal should stand out in the opposite-sign (OS) dimuon channel in the case of the HS model; the DR3 model will require higher integrated luminosity to yield a signal in the OS dimuon channel. This region may also be probed by the Tevatron with $5-10 \mathrm{fb}^{-1}$ of data, if a corresponding search in the multi- $b+E_{T}^{\text {miss }}$ channel is performed. With higher integrated luminosities of $\sim 1 \mathrm{fb}^{-1}$, using $E_{T}^{\text {miss }}$ plus a large multiplicity of $b$-jets, LHC should be able to discover Yukawa-unified SUSY with $m_{\tilde{g}} \lesssim 630 \mathrm{GeV}$. Thus, the year 1 LHC reach for Yukawa-unified SUSY should be enough to either claim a discovery of the gluino, or to very nearly rule out this class of models, since higher values of $m_{\tilde{g}}$ lead to rather poor Yukawa unification.

KeYWORDS: Supersymmetry Phenomenology

ArXiv EPRINT: 0911.4739 


\section{Contents}

1 Introduction 1

$2 \quad$ HS and DR3 model lines $\quad 6$

2.1 Model lines 6

$\begin{array}{lll}2.2 & \text { HS and DR3 production cross sections } & 7\end{array}$

2.3 Sparticle branching fractions in the HS and DR3 model lines $\quad 7$

$\begin{array}{llr}3 & \text { Event simulation } & 10\end{array}$

4 Early searches without $E_{T}^{\text {miss }}$ or electron ID 11

$\begin{array}{lll}4.1 & \text { Multi } b \text {-jet signal } & 12\end{array}$

$\begin{array}{lll}4.2 & \text { Dimuon channels } & 14\end{array}$

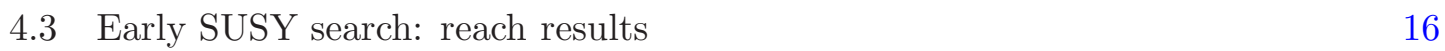

5 Analysis including $E_{T}^{\text {miss }}$ cut and electron ID 18

$\begin{array}{llr}5.1 & \text { Multi } b \text {-jet }+E_{T}^{\text {miss }} \text { channel } & 19\end{array}$

$\begin{array}{ll}5.2 \text { Multi-lepton channels } & 20\end{array}$

5.3 Jets plus $Z \rightarrow \ell \bar{\ell}+E_{T}^{\text {miss }}$ signal 23

5.4 LHC reach for Yukawa-unified SUSY using $E_{T}^{\text {miss }}$ and $e$ ID 23

5.5 Differentiating the HS and DR3 models 26

6 Comparison with CDF/CMS multijets $+E_{T}^{\text {miss }}$ channel 27

$\begin{array}{llr}7 & \text { Conclusions } & 29\end{array}$

\section{Introduction}

Grand unified theories (GUTs) find a welcome inclusion of supersymmetry (SUSY) into their structure in that SUSY tames the gauge hierarchy problem via the well-known cancellation of quadratic divergences $[1,2]$. In particular, the GUT group $\mathrm{SO}(10)$ is highly motivated in that it allows for- in addition to gauge unification- the unification of all the matter superfields of each generation into the 16-dimensional spinor representation [3]. The matter unification only works if the 15 matter superfields of the Minimal Supersymmetric Standard Model (MSSM) are augmented by a SM gauge singlet superfield $\hat{N}_{i}^{c}$ which contains a right-hand neutrino (RHN) field. The presence of RHN fields is essential to describe data from the past decade on neutrino mass and flavor oscillations; in particular a Majorana mass term near the GUT scale, needed to implement see-saw neutrino masses [6-8], should be generated by the breakdown of $\mathrm{SO}(10)$ gauge symmetry. In addition to gauge and matter unification, in the simplest SO(10) SUSY GUT models- wherein both MSSM 
Higgs doublets reside in a 10 of $\mathrm{SO}(10)$ - one expects Yukawa coupling unification in the third generation: $f_{t}=f_{b}=f_{\tau}\left(=f_{\nu_{\tau}}\right)$ at $M_{\mathrm{GUT}}$.

Recently, a variety of studies have examined the $\operatorname{MSSM}(+\mathrm{RHN})$ to check whether the measured values of gauge couplings and third generation fermion masses do indeed allow for $t-b-\tau$ Yukawa coupling unification [9-42]. Essential to the calculation is the inclusion of 2-loop renormalization group equations [43] (RGEs) and inclusion of weak scale threshold corrections $[14,44-46]$ which occur due to the MSSM $\rightarrow$ SM transition in effective field theories. These threshold corrections imply that Yukawa coupling unification depends on the entire spectrum of SUSY particles, since the SUSY particles enter the various $t, b$ and $\tau$ self-energy diagrams $[14,44-46]$.

Assuming universal boundary conditions at the GUT scale, the parameter space of SO(10)-motivated SUSY consists of

$$
m_{1 / 2}, m_{16}, m_{10}, M_{D}^{2}, A_{0}, \tan \beta, \operatorname{sign}(\mu),
$$

where $m_{1 / 2}$ is the common gaugino mass at $M_{\mathrm{GUT}}, m_{16}$ is the common GUT mass of all matter scalars, $m_{10}$ is that of the Higgs soft terms, and $M_{D}^{2}$ parametrizes potential splittings in the GUT scale Higgs (and possibly matter scalar) soft terms. Such splittings are expected to arise from the breaking of the $\mathrm{SO}(10)$. It has been found that $t-b-\tau$ Yukawa coupling unification can occur in the MSSM within this setup, but only for very restricted forms of the soft SUSY breaking (SSB) parameters at $M_{\mathrm{GUT}}$. These include, for the case of $\mu>0$ :

- $A_{0}^{2}=2 m_{10}^{2}=4 m_{16}^{2}$,

- $m_{16} \sim 5-15 \mathrm{TeV}$,

- $m_{1 / 2} \ll m_{16}$,

- $\tan \beta \sim 50$.

These boundary conditions were found in ref. [47-51] to give rise to an inverted scalar mass hierarchy (IMH), wherein first/second generation scalars end up with masses $\sim 10 \mathrm{TeV}$, while third generation scalars, Higgs scalars $A, H$ and $H^{ \pm}$and $\mu$ are of order $\sim 1-2 \mathrm{TeV}$.

A problem with the IMH scheme is that it is inconsistent with radiative electroweak symmetry breaking (REWSB), unless the Higgs soft terms are split at $M_{\mathrm{GUT}}$ [52]: $m_{H_{u}}^{2}<$ $m_{H_{d}}^{2}$, thus giving $m_{H_{u}}^{2}$ a head start over $m_{H_{d}}^{2}$ in its running towards the weak scale. ${ }^{1}$ Such splitting naturally occurs due to $D$-term (DT) contributions to all scalar masses arising from the breakdown of $\mathrm{SO}(10)$. However, applying the splitting to only the Higgs sector ("just-so" Higgs splitting, HS)

$$
m_{H_{u, d}}^{2}=m_{10}^{2} \mp 2 M_{D}^{2} \quad(\text { HS model })
$$

results in better accuracy of Yukawa unification as compared to full DT splitting. Recently, it has been shown that DT splitting, combined with the running effect of the neutrino

\footnotetext{
${ }^{1}$ This can be different in non-universal models, see [37, 40, 41].
} 

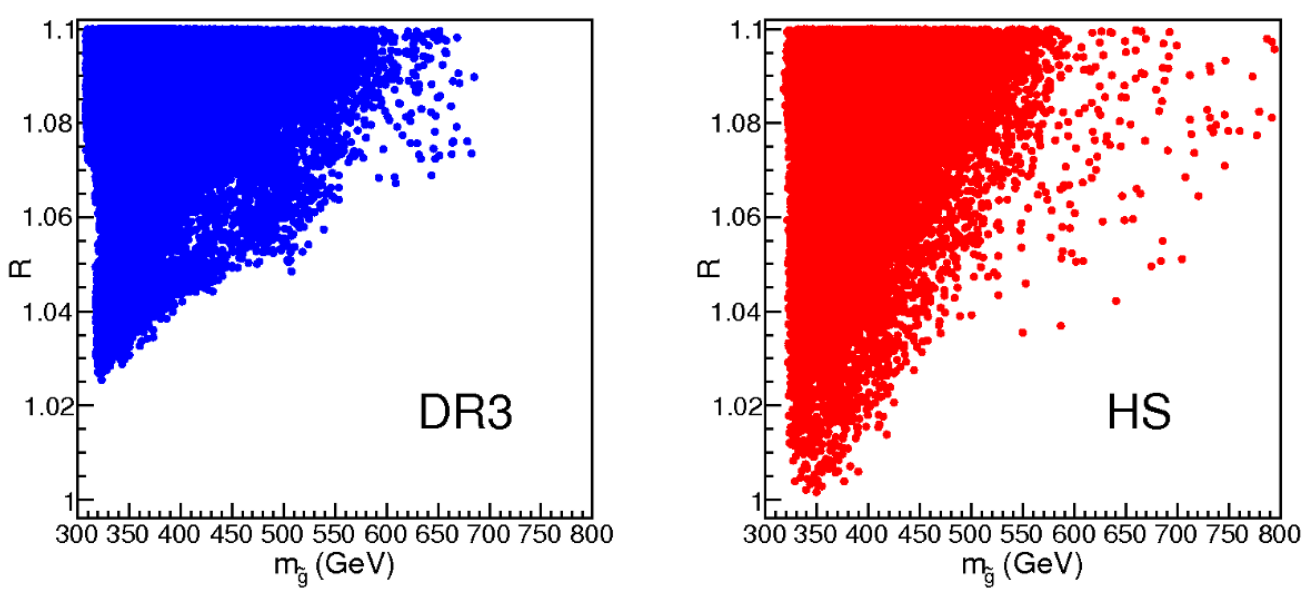

Figure 1. Scatter plot of Yukawa unified models in the $R$ vs. $m_{\tilde{g}}$ plane, for solutions in the DR3 model (blue) and the HS model (red).

Yukawa coupling $f_{\nu_{\tau}}$ and a small mass splitting between first/second versus third generation scalars (the DR3 model) can allow for Yukawa coupling unification to a few percent [42].

Both the HS and DR3 schemes lead to sparticle mass spectra characterized by

- $m_{\tilde{q}, \tilde{\ell}}(1,2) \sim 10 \mathrm{TeV}$,

- $m_{\tilde{q}, \tilde{\ell}}(3)$ and $\mu \sim 1-3 \mathrm{TeV}$

- $m_{\tilde{g}} \sim 300-500 \mathrm{GeV}$,

- $m_{\widetilde{W}_{1}, \widetilde{Z}_{2}} \sim 100-180 \mathrm{GeV}$,

- $m_{\widetilde{Z}_{1}} \sim 50-90 \mathrm{GeV}$.

Figure 1 shows the location of a large number of Yukawa-unified models in the $R$ vs. $m_{\tilde{g}}$ plane, for the HS model (red dots) and the DR3 model (blue dots) obtained through a Markov Chain Monte Carlo (MCMC) scan of the parameter space (for details, see [42]). Here, the degree of Yukawa unification is quantified as

$$
R=\frac{\max \left(f_{t}, f_{b}, f_{\tau}\right)}{\min \left(f_{t}, f_{b}, f_{\tau}\right)}
$$

where $f_{t}, f_{b}$ and $f_{\tau}$ are the top, bottom and tau Yukawa couplings, respectively, evaluated at $Q=M_{\mathrm{GUT}}$. In the DR3 case, if we require $R<1.05$, then $m_{\tilde{g}} \lesssim 450 \mathrm{GeV}$. In the HS model, while $m_{\tilde{g}} \sim 300-500 \mathrm{GeV}$ is favored for low $R<1.05$ solutions, it is possible (but not likely) to have occassional models with $m_{\tilde{g}}$ as large as $\sim 700 \mathrm{GeV}$.

In models with the above listed superpartner spectrum and a bino-like $\widetilde{Z}_{1}$ state, the neutralino relic density is computed to be $\sim 10^{2}-10^{4}$ times the measured abundance [36, 38], and the models are seemingly excluded. However, if one invokes the Peccei-Quinn solution to the strong $C P$ problem [53-63], then an axion/axino supermultiplet is expected 
in the theory [64-66]. With an axino of mass $m_{\tilde{a}} \sim 1 \mathrm{MeV}$ the neutralinos will decay via $\widetilde{Z}_{1} \rightarrow \tilde{a} \gamma$, which greatly reduces the dark matter density by a large factor: $m_{\tilde{a}} / m_{\widetilde{Z}_{1}}$. Cold dark matter $(\mathrm{CDM})$ solutions can be found consisting of mainly cold axions and thermally produced axinos, with a small component of warm axinos arising from $\widetilde{Z}_{1} \rightarrow \gamma \tilde{a}$ decay, which occurs on time scales of order 1 sec. Since $m_{16} \sim 10 \mathrm{TeV}$, and we expect $m_{16} \sim m_{\tilde{G}}$, the axion/axino CDM scenario allows for a solution to the gravitino BBN problem, and can generate re-heat temperatures $T_{R} \sim 10^{6}-10^{9} \mathrm{GeV}$, which can allow for baryogenesis mechanisms such as non-thermal [67-69] or Affleck-Dine [70, 71] leptogenesis to occur [72].

Since the value of $m_{\tilde{g}}$ is so low in Yukawa-unified SUSY models, we expect the whole scenario to soon be tested at the CERN LHC. ${ }^{2}$ LHC has already turned on in Fall, 2009. As time progresses, the centre-of-mass energy $\sqrt{s}$ will be increased into the $\sim 7 \mathrm{TeV}$ regime. An integrated luminosity of $0.1-1 \mathrm{fb}^{-1}$ is expected to be collected. ${ }^{3}$ Earlier work on Yukawa-unified SUSY at LHC with $\sqrt{s}=14 \mathrm{TeV}$ showed the model to be easily testable at LHC [74]. The LHC SUSY events should be characterized by gluino pair production followed by three-body decays to states including a high multiplicity of $b$-jets. In addition, opposite-sign dileptons with mass between $40-80 \mathrm{GeV}$ (i.e. between the $\gamma$ and $Z$ peaks) may be evident.

In the intervening past year, while LHC recovered from an unfortunate incident involving faulty circuits and quenched magnets, the experiments have been measuring millions of cosmic muon events. This cosmic data has allowed them to fine-tune their detector response to muons, and to make great strides in alignment of detector elements. We expect thus that isolated muons, jets and $b$-jets should be readily measurable very early on during LHC running. Reliable electron identification ( $e$ ID) requires distinguishing mainly EM showers from mixed EM/HAD showers, and may take more time to properly establish. Even more so, reliable $E_{T}^{\text {miss }}$ measurement may require additional time to establish, since its measurement relies on a complete knowledge of all detector elements: such a complete knowledge will not likely be gained until calibration on a variety of well-known SM processes is made.

In this paper, we expand upon the analysis presented in ref. [74], and address several new issues:

- We focus on the LHC potential to discover or rule out Yukawa-unified SUSY during year 1 of running. ${ }^{4}$ To this end, we calculate signal and background production rates for the LHC turn-on energy of $\sqrt{s}=7 \mathrm{TeV}$ rather than the maximal collider energy of $\sqrt{s}=14 \mathrm{TeV}$ used earlier. Part of the effect of LHC turn-on at lower than expected energies can be gleaned from figure 2 , where we plot $\sigma(p p \rightarrow \tilde{g} \tilde{g} X)$ vs. collider energy $\sqrt{s}$, for $m_{\tilde{g}}=300,400$ and $500 \mathrm{GeV}$, while taking $m_{\tilde{q}}=10 \mathrm{TeV}$. We show both LO and NLO QCD results as derived from Prospino [84]. For $m_{\tilde{g}}=400 \mathrm{GeV}$, LHC operating at $\sqrt{s}=7 \mathrm{TeV}$ yields a cross section of $\sigma \sim 10^{4} \mathrm{fb}$. It is expected that, after about $0.1 \mathrm{fb}^{-1}$ of integrated luminosity, the LHC will move up in energy to the $\sqrt{s} \sim 10 \mathrm{TeV}$ regime, where $\sigma \sim 3 \times 10^{4} \mathrm{fb}$. Ultimately, the LHC should

\footnotetext{
${ }^{2}$ Indeed experiments at the Fermilab Tevatron collider may also probe up to $m_{\tilde{g}} \sim 400-430 \mathrm{GeV}$ [73].

${ }^{3}$ The quoted physics data to be collected at $7 \mathrm{TeV}$ as of November 2009 is $0.2 \mathrm{fb}^{-1}$.

${ }^{4}$ Some additional analyses of early physics prospects at LHC are contained in refs. [75-83].
} 


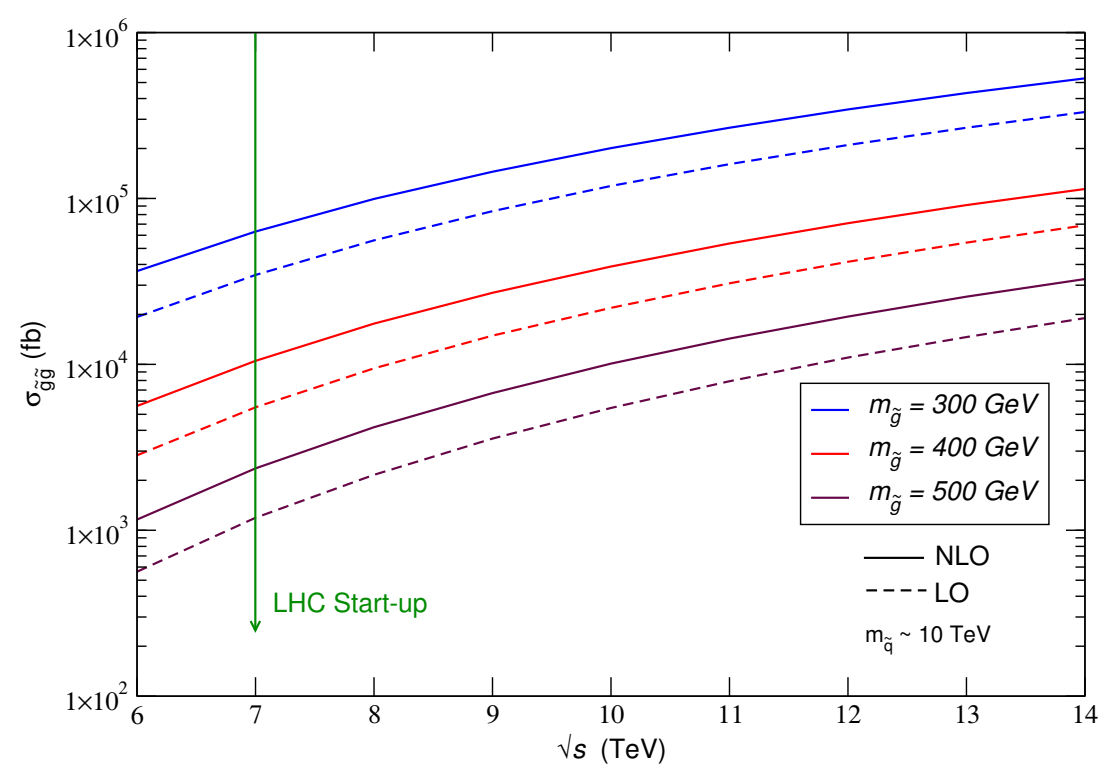

Figure 2. Total cross-section for gluino pair production with $m_{\tilde{q}}=10 \mathrm{TeV}$ versus LHC collider energy $\sqrt{s}$, for $m_{\tilde{g}}=300,400$ and $500 \mathrm{GeV}$.

move up to its design energy of $\sqrt{s}=14 \mathrm{TeV}$, where the cross section increases to $\sim 10^{5} \mathrm{fb}$. Various background rates will also change accordingly. In this paper, we take a conservative approach, and evaluate all signal and background cross sections at $\sqrt{s}=7 \mathrm{TeV}$. Increasing the beam energy beyond $7 \mathrm{TeV}$ should only increase the SUSY reach projections which we calculate here.

- We include as well many more background subprocesses than before, including the effect of many $2 \rightarrow 3$ and $2 \rightarrow 4$ body subprocesses.

- We particularly hone in on what LHC can accomplish with very low integrated luminosity. After turn-on, some time will be required to examine detector response to well-known SM processes like $W, Z$ and $t \bar{t}$ production. To be able to use the classic SUSY signature of jets $+E_{T}^{\text {miss }}$ production, the measurement of $E_{T}^{\text {miss }}$ - which depends on a knowledge of the entire detector response- will be required. However, in ref. [80-83], it is pointed out that LHC experiments can examine multi-jet + isolated multi-muon events in lieu of jets $+E_{T}^{\text {miss }}$ events as a gain for signal over background. We find that for very low integrated luminosity, using either large isolated muon multiplicity, or large $b$-jet multiplicity, allows $m_{\tilde{g}}$ values of up to $400 \mathrm{GeV}$ to be probed with just $0.2 \mathrm{fb}^{-1}$ of integrated luminosity. (Note that we expect a similar reach for the Tevatron in the $\geq 2-3 b$-jets $+E_{T}^{\text {miss }}$ channel with $5-10 \mathrm{fb}^{-1}$ [73].)

- We also discuss the case when $E_{T}^{\text {miss }}$ measurements and $e$ ID are established. Here we find that LHC can explore $m_{\tilde{g}}$ values as high as $\sim 630 \mathrm{GeV}$ with $1 \mathrm{fb}^{-1}$ of integrated luminosity. Thus, during year 1 the LHC may well be able to either discover or very nearly rule out Yukawa-unified SUSY. 
The paper is organized as follows. We first establish in section 2 two Yukawa-unified model lines: one in the HS model and one in the DR3 model. We also examine general features in sparticle production and decay for these model lines. In section 3, we present some technical details of our signal and background calculations. In section 4, we present expectations for early SUSY searches in the multi $b$-jets channel ${ }^{5}$ without using $E_{T}^{\text {miss }}$ cuts. We also examine rates for early multi-muon production plus jets without using $E_{T}^{\text {miss }}$. We find that the HS and DR3 models may be distinguishable by measuring the ratio of OS dilepton events to multi $b$-jet events, since both models produce multi- $b$-jets at a similar rate. However, while OS dimuons from $\widetilde{Z}_{2}$ decay are abundant in the HS model, they are relatively scarce in the DR3 model. In section 5.4, we move beyond the $0.1 \mathrm{fb}^{-1}$ level, and calculate the LHC reach for the two model lines using as well $E_{T}^{\text {miss }}$ and $e$ ID for $1 \mathrm{fb}^{-1}$ of integrated luminosity. In this case, the $5 \sigma$ LHC reach should extend to $m_{\tilde{g}} \sim 630 \mathrm{GeV}$, enough to cover the bulk of parameter space of these simple Yukawa-unified models. In section 7 , we present our conclusions.

\section{$2 \quad$ HS and DR3 model lines}

\subsection{Model lines}

Using the parameter space in eq. 1.1, ref. [72] found a large number of SUSY spectral solutions with good Yukawa coupling unification in the HS model. We adopt Point B with $R=1.02$ of this paper as a Yukawa-unified benchmark point, and label it as HSb. The $\mathrm{HSb}$ input parameters and mass spectra are listed in table 1.

To construct a HS model line, we keep most of the above parameters fixed, but allow $m_{1 / 2}$ to vary. This keeps the Yukawa-unification generally low, but allows us to vary $m_{\tilde{g}} \sim 3.5 m_{\widetilde{Z}_{2}} \sim 7 m_{\widetilde{Z}_{1}}$ continuously. We plot the value of $R$ versus $m_{\tilde{g}}$ in figure 3 . We see that at low $m_{\tilde{g}}(\sim 325 \mathrm{GeV}), R<1.03$, while as $m_{\tilde{g}}$ increases, Yukawa unification gets worse until $m_{\tilde{g}} \sim 700 \mathrm{GeV}$, where we find $R \sim 1.13$.

We also adopt from ref. [42] a DR3 model line, labelled as DR3b, with parameters in table 1 . where $m_{16}(1,2,3)$, is the scalar mass for the 1st, 2nd and 3th generations and $M_{N_{3}}, f_{\nu_{\tau}}, A_{\nu_{\tau}}$ and $m_{\tilde{v}_{R 3}}$ are the right-handed neutrino mass, Yukawa coupling, $A$-term and the scalar mass for the sneutrino. We construct a DR3 model line by again keeping most parameters fixed, but letting $m_{1 / 2}$ to vary. In the DR3 model line, we find $R \sim 1.03$ for $m_{\tilde{g}} \sim 325 \mathrm{GeV}$, while $R$ increases to $\sim 1.15$ for $m_{\tilde{g}} \sim 700 \mathrm{GeV} .^{6}$

Due to the heavy scalar masses in the HS and DR3 model lines, the scalars essentially decouple at LHC energies. What results is a low energy effective theory where only $\tilde{g}$, $\widetilde{Z}_{1,2}$ and $\widetilde{W}_{1}^{ \pm}$are the new physics matter states. In the next two sections we discuss the production cross-sections and decay rates for these states.

\footnotetext{
${ }^{5}$ Earlier work emphasizing the utility of the presence of $b$-jets in SUSY events was provided in refs. [74, 85$88]$.

${ }^{6}$ Although figure 1 shows that for some special choice of the parameters lower values of $R$ can be obtained for $m_{\tilde{g}} \sim 700$, the model lines chosen here represent the general behavior of the bulk of parameter space.
} 


\begin{tabular}{|lcc|}
\hline parameter & HSb & DR3b \\
\hline$m_{16}(1,2)$ & 10000 & 11805.6 \\
$m_{16}(3)$ & 10000 & 10840.1 \\
$m_{10}$ & 12053.5 & 13903.3 \\
$M_{D}$ & 3287.1 & 1850.6 \\
$m_{1 / 2}$ & 43.9442 & 27.414 \\
$A_{0}$ & -19947.3 & -22786.2 \\
$\tan \beta$ & 50.398 & 50.002 \\
$R$ & 1.025 & 1.027 \\
$\mu$ & 3132.6 & 2183.4 \\
\hline$m_{\tilde{g}}$ & 351.2 & 321.4 \\
$m_{\tilde{u}_{L}}$ & 9972.1 & 11914.2 \\
$m_{\tilde{t}_{1}}$ & 2756.5 & 2421.6 \\
$m_{\tilde{b}_{1}}$ & 3377.1 & 1359.5 \\
$m_{\tilde{e}_{R}}$ & 10094.7 & 11968.5 \\
$m_{\widetilde{W}_{1}}$ & 116.4 & 114.5 \\
$m_{\widetilde{Z}_{2}}$ & 113.8 & 114.2 \\
$m_{\widetilde{Z}_{1}}$ & 49.2 & 46.5 \\
$m_{A}$ & 1825.9 & 668.3 \\
$m_{h}$ & 127.8 & 128.6 \\
\hline$\sigma($ tot. $)$ & $18030 \mathrm{fb}$ & $24747 \mathrm{fb}$ \\
\hline
\end{tabular}

Table 1. Masses in GeV units and parameters for Yukawa-unified benchmark points HSb [72] and DR3b [42]. For the DR3 model, we use $M_{N_{3}}=10^{13} \mathrm{GeV}$. We also show the total cross section for LHC collisions at $\sqrt{s}=7 \mathrm{TeV}$.

\subsection{HS and DR3 production cross sections}

We plot in figure 4 the leading order $\tilde{g} \tilde{g}, \widetilde{W}_{1} \widetilde{Z}_{2}$ and $\widetilde{W}_{1}^{+} \widetilde{W}_{1}^{-}$production cross sections as a function of $m_{\tilde{g}}$ for collider energy $\sqrt{s}=7 \mathrm{TeV}$. From the figure, we see that gluino pair production is dominant up to $m_{\tilde{g}} \sim 520 \mathrm{GeV}$ (at NLO, it dominates up to $m_{\tilde{g}} \sim$ $560 \mathrm{GeV}$ ), with cross sections typically greater than $10^{3} \mathrm{fb}$, and in excess of $10^{4} \mathrm{fb}$ in the lower gluino mass range. Thus, even with integrated luminosities as low as $0.1 \mathrm{fb}^{-1}$, we expect hundreds of gluino pair events in the upcoming LHC year 1 physics data sample for the HS and DR3 models.

Gluino pair production cross sections at the Fermilab Tevatron collider show a large increase in rate as $m_{\tilde{q}}$ increases [73]. This is due to suppression of negative interference terms in the $q \bar{q} \rightarrow \tilde{g} \tilde{g}$ subprocess cross section. At the LHC, gluino pair production for $m_{\tilde{g}} \sim 300-500 \mathrm{GeV}$ is dominated instead by the $g g \rightarrow \tilde{g} \tilde{g}$ subprocess, which is independent of $m_{\tilde{q}}$. Thus, $\tilde{g} \tilde{g}$ cross sections show only a slight $(\sim 10-20 \%)$ increase with increasing $m_{\tilde{q}}$ at the LHC.

\subsection{Sparticle branching fractions in the HS and DR3 model lines}

Since $m_{\tilde{g}} \ll m_{\tilde{q}}$ in the HS or DR3 model lines, we will get dominant gluino decays into three-body modes. The gluino branching ratios will be largely model dependent, but since 


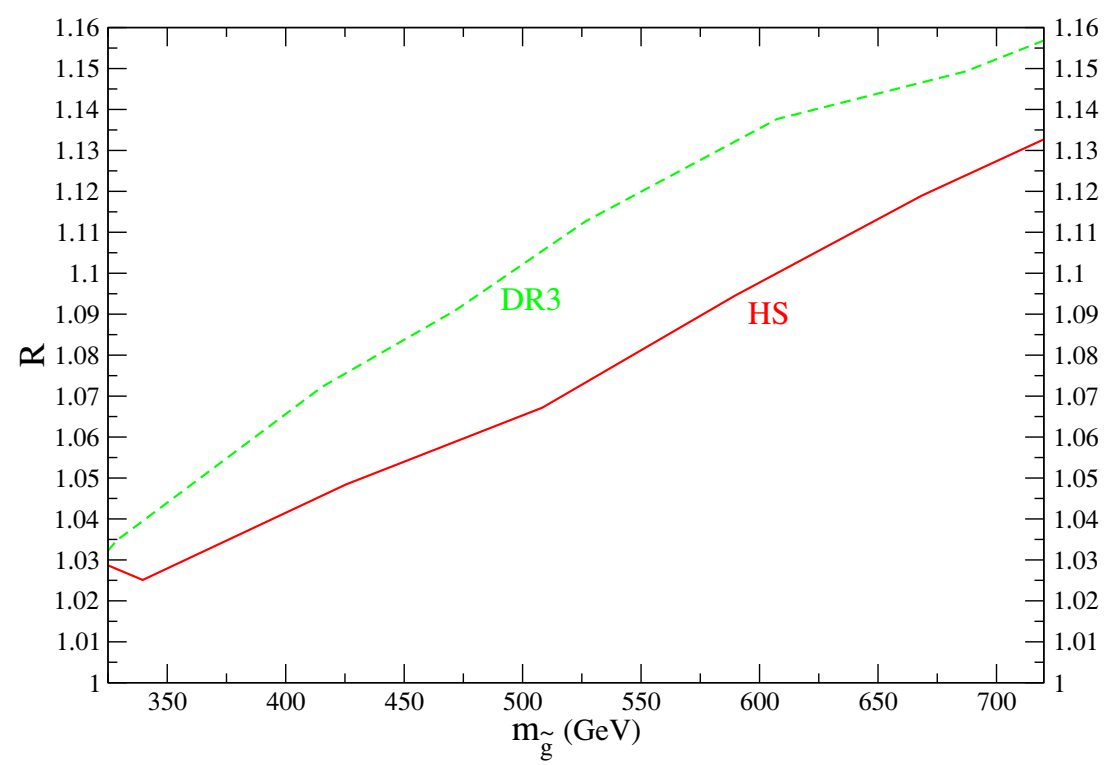

Figure 3. Degree of Yukawa unification $R$ (see eq. 1.3) for the models HS and DR3 as a function of the gluino mass. The model parameters are the same as in table 1 , but with $m_{1 / 2}$ varying from 30 to $180 \mathrm{GeV}$.

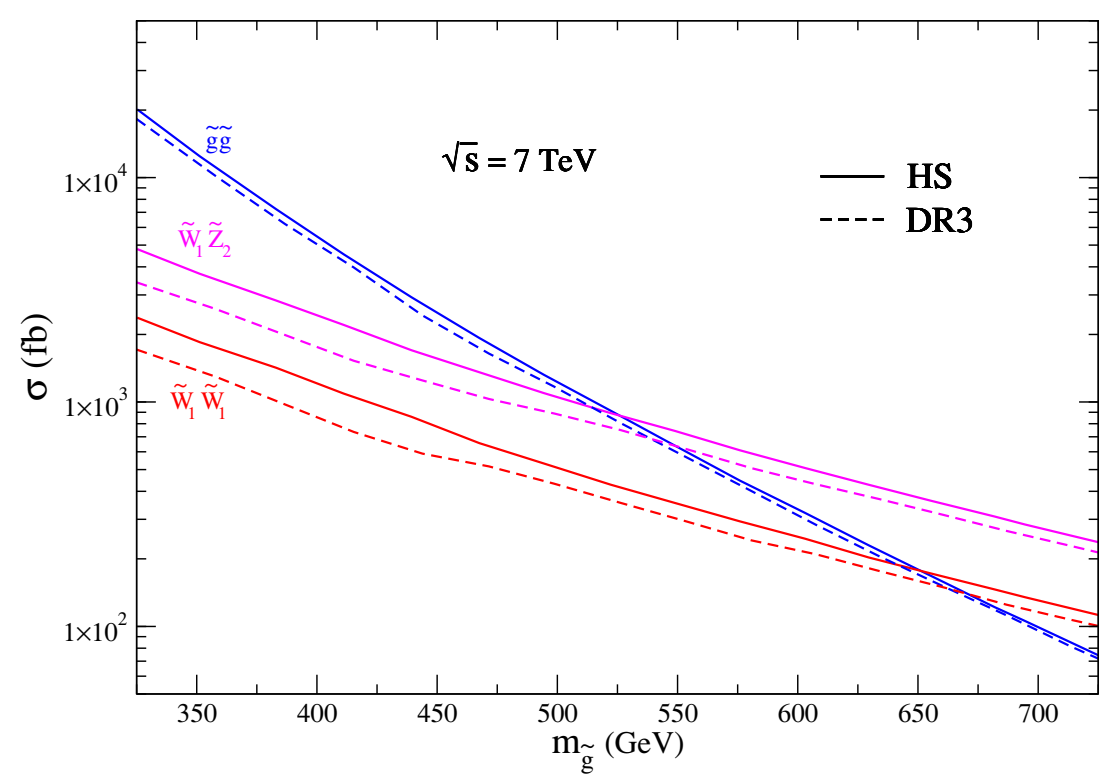

Figure 4. Leading order total cross-sections for sparticle production in the HS model (solid) and DR3 model (dashed) as a function of the gluino mass for $p p$ collisions at $\sqrt{s}=7 \mathrm{TeV}$. The model parameters are the same as in table 1 but with $m_{1 / 2}$ varying from 30 to $180 \mathrm{GeV}$.

$\tilde{t}_{i}$ and $\tilde{b}_{i}$ are always the lightest squarks, and $\tan \beta$ is large [89, 90], the decays will mostly be restricted to the following channels:

- $\tilde{g} \rightarrow \widetilde{Z}_{i}+b \bar{b}, i=1,2$

- $\tilde{g} \rightarrow \widetilde{Z}_{1}+t \bar{t}$ 

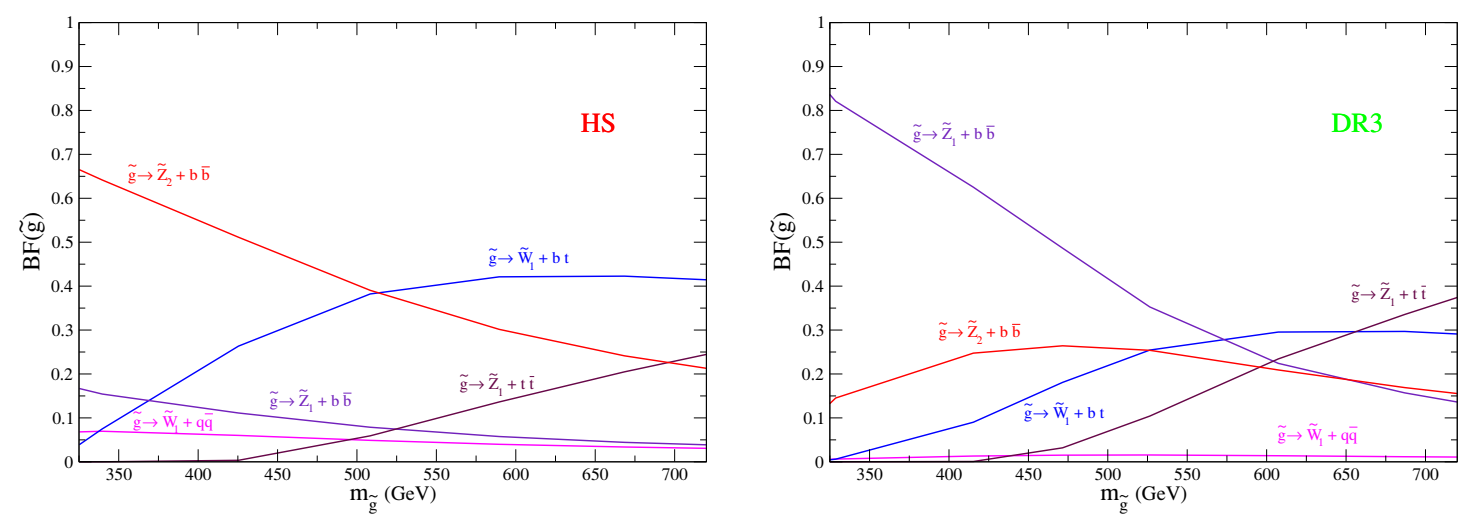

Figure 5. Gluino branching ratios for the HS and DR3 model-lines as a function of the gluino mass. The model parameters are the same as in table 1 , but with $m_{1 / 2}$ varying from 30 to $180 \mathrm{GeV}$.

- $\tilde{g} \rightarrow \widetilde{W}_{1}^{-} \bar{b} t$ or $\widetilde{W}_{1}^{+} b \bar{t}$.

The general feature $m_{\tilde{g}} \ll m_{\tilde{q}}$ is common to both the HS and DR3 models, since it relies mostly on the fact that $m_{1 / 2} \ll m_{16}$. However, the inclusion of the $D$-term splitting for all matter scalars in the DR3 model pushes $m_{\tilde{b}_{R}}$ to lower values, when compared to the HS model, where $m_{\tilde{b}_{L}} \sim m_{\tilde{b}_{R}} \cdot{ }^{7}$ As a result we have:

- DR3: $\tilde{b}_{1} \sim \tilde{b}_{R}$ and $m_{\tilde{b}_{1}}<m_{\tilde{b}_{2}}$

- HS: $\tilde{b}_{1} \sim \tilde{b}_{L}$ and $m_{\tilde{b}_{1}} \sim m_{\tilde{b}_{2}}$

Now, since $\widetilde{Z}_{2}$ is wino-like in both models, it just couples to left-squarks, what suppresses the $\tilde{g} \rightarrow \widetilde{Z}_{2}+b \bar{b}$ decay in the DR3 model and favors it in the HS case. This behavior is shown in figure 5 , where the main gluino branching ratios for both models are plotted as a function of the gluino mass. From figure 5 it can also be seen that- in the HS modelonce $m_{\tilde{g}} \gg m_{t}+m_{\widetilde{W}_{1}}$ the $\tilde{g} \rightarrow \widetilde{W}_{1}^{-} \bar{b} t+$ c.c. channel starts to dominate, since $m_{\tilde{t}_{1}}<m_{\tilde{b}_{1}}$ $\left(\right.$ for $m_{\tilde{g}}>500 \mathrm{GeV}$ ). We can also see that $\tilde{g} \rightarrow \widetilde{Z}_{1} t \bar{t}$ becomes relevant for heavy gluinos $\left(m_{\tilde{g}}>600 \mathrm{GeV}\right)$ and it is enhanced in the DR3 model, where $\tilde{t}_{1}$ is usually lighter than in the HS model.

From figure 5 we see that, for the HSb benchmark case:

- $B R\left(\tilde{g} \rightarrow \widetilde{Z}_{2}+b \bar{b}\right)=63 \%$

- $B R\left(\tilde{g} \rightarrow \widetilde{Z}_{1}+b \bar{b}\right)=15 \%$

- $B R\left(\tilde{g} \rightarrow \widetilde{W}_{1}+b t\right)=9 \%$.

On the other hand, the DR3b point has:

- $B R\left(\tilde{g} \rightarrow \widetilde{Z}_{2}+b \bar{b}\right)=11 \%$

${ }^{7}$ The stop masses and mixing are basically the same in both models, since the $D$-term splitting is equal for both $\tilde{t}_{R}$ and $\tilde{t}_{L}$. 

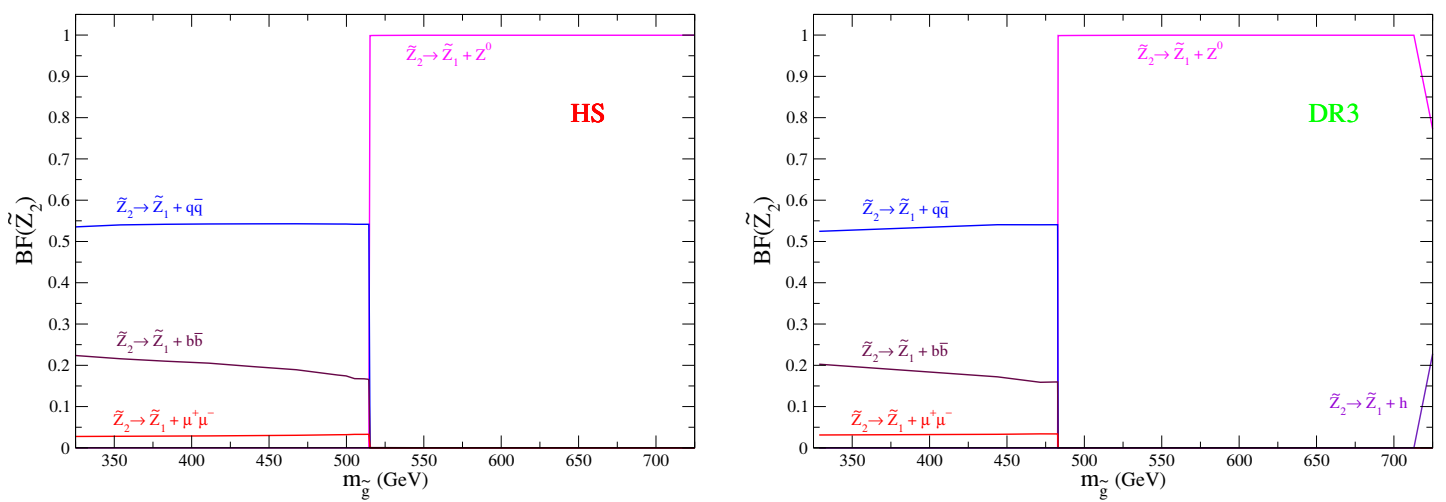

Figure 6. $\widetilde{Z}_{2}$ branching ratios for the HS and DR3 model-lines as a function of the gluino mass. The model parameters are the same as in table 1 , but with $m_{1 / 2}$ varying from 30 to $180 \mathrm{GeV}$.

- $B R\left(\tilde{g} \rightarrow \widetilde{Z}_{1}+b \bar{b}\right)=86 \%$

- $B R\left(\tilde{g} \rightarrow \widetilde{W}_{1}+b t\right)=0.3 \%$.

The $\widetilde{Z}_{2}$ and $\widetilde{W}_{1}$ are expected to decay via three body modes:

- $\widetilde{Z}_{2} \rightarrow \widetilde{Z}_{1} f \bar{f}$

- $\widetilde{W}_{1}^{ \pm} \rightarrow \widetilde{Z}_{1} f \bar{f}^{\prime}$,

where the decays are dominated by the intermediate virtual $W^{*}$ and $Z^{*}$ diagrams. If $m_{\tilde{g}} \gtrsim 500 \mathrm{GeV}$, then the two-body modes $\widetilde{W}_{1} \rightarrow \widetilde{Z}_{1} W$ and $\widetilde{Z}_{2} \rightarrow \widetilde{Z}_{1} Z$ will turn on.

Putting all segments of the cascade decays together, we expect the HSb signal to be rich in $b$-jets and opposite-sign/same-flavor (OS/SF) isolated dileptons coming from $\widetilde{Z}_{2} \rightarrow \widetilde{Z}_{1} \ell \bar{\ell}$, with a small rate of SS dileptons coming from $\tilde{g} \rightarrow \widetilde{W}_{1} q \bar{q}^{\prime}$ followed by $\widetilde{W}_{1} \rightarrow \ell \nu_{\ell} \widetilde{Z}_{1}$ decay. For the DR3b point, we expect the signal to be rich in $b$-jets with a harder $E_{T}^{\text {miss }}$ spectrum (when compared to HSb) due to the direct gluino decay to $\widetilde{Z}_{1}$, but with small rates in the multilepton channels.

\section{Event simulation}

In order to study the discovery potential of the LHC at $\sqrt{s}=7 \mathrm{TeV}$ we used AlpGen [91] and MadGraph [92] to generate the background hard scattering events and Pythia [93] for the subsequent showering and hadronization. Table 2 lists the $2 \rightarrow n$ subprocesses included in this study where jets $=u, d, s, c$ and $g$. For all the processes involving multiple jets, the MLM matching algorithm [91] was used to avoid double counting. All the above processes were generated at LO, but a relatively low renormalization and factorization scale $(Q=\sqrt{\hat{s}} / 6)$ was used to bring the total cross-sections closer to their NLO values (for more details see ref. [83]). The signal events were generated using Isajet 7.79 [94, 95].

A toy detector simulation is then employed with calorimeter cell size $\Delta \eta \times \Delta \phi=$ $0.05 \times 0.05$ and $-5<\eta<5$. The HCAL (hadronic calorimetry) energy resolution is taken to be $80 \% / \sqrt{E}+3 \%$ for $|\eta|<2.6$ and FCAL (forward calorimetry) is $100 \% / \sqrt{E}+5 \%$ for 


\begin{tabular}{|l|c|c|c|}
\hline SM process & Generator & $\begin{array}{c}\text { Cross } \\
\text { section }\end{array}$ & $\begin{array}{c}\text { number of } \\
\text { events }\end{array}$ \\
\hline QCD: 2,3 and 4 jets $\left(p_{T}>40 \mathrm{GeV}\right)$ & AlpGen & $3.0 \times 10^{9} \mathrm{fb}$ & $13 \mathrm{M}$ \\
$t \bar{t}: t \bar{t}+0,1$ and 2 jets & AlpGen & $1.6 \times 10^{5} \mathrm{fb}$ & $5 \mathrm{M}$ \\
$b \bar{b}: b \bar{b}+0,1$ and 2 jets & AlpGen & $8.8 \times 10^{7} \mathrm{fb}$ & $91 \mathrm{M}$ \\
$Z+$ jets: $Z / \gamma(\rightarrow l \bar{l}, \nu \bar{\nu})+0,1,2$ and 3 jets & AlpGen & $8.8 \times 10^{6} \mathrm{fb}$ & $13 \mathrm{M}$ \\
$W+$ jets: $W^{ \pm}(\rightarrow l \nu)+0,1,2$ and 3 jets & AlpGen & $1.8 \times 10^{7} \mathrm{fb}$ & $19 \mathrm{M}$ \\
$Z+t \bar{t}: Z / \gamma(\rightarrow l \bar{l}, \nu \bar{\nu})+t \bar{t}+0,1$ and 2 jets & AlpGen & $53 \mathrm{fb}$ & $0.6 \mathrm{M}$ \\
$Z+b \bar{b}: Z / \gamma(\rightarrow l \bar{l}, \nu \bar{\nu})+b \bar{b}+0,1$ and 2 jets & AlpGen & $2.6 \times 10^{3} \mathrm{fb}$ & $0.3 \mathrm{M}$ \\
$W+b \bar{b}: W^{ \pm}(\rightarrow l \nu)+b \bar{b}+0,1$ and 2 jets & AlpGen & $6.4 \times 10^{3} \mathrm{fb}$ & $9 \mathrm{M}$ \\
$t \bar{t} t \bar{t}$ & MadGraph & $0.6 \mathrm{fb}$ & $1 \mathrm{M}$ \\
$t \bar{t} b \bar{b}$ & MadGraph & $1.0 \times 10^{2} \mathrm{fb}$ & $0.2 \mathrm{M}$ \\
$b \bar{b} b \bar{b}$ & MadGraph & $1.1 \times 10^{4} \mathrm{fb}$ & $0.07 \mathrm{M}$ \\
\hline
\end{tabular}

Table 2. Background processes included in this study, their cross sections and number of generated events.

$|\eta|>2.6$, where the two terms are combined in quadrature. The ECAL (electromagnetic calorimetry) energy resolution is assumed to be $3 \% / \sqrt{E}+0.5 \%$. We use the Isajet jet finding algorithm (cone type) to group the hadronic final states into jets. The jets and isolated lepton definitions are as follow:

- Jets are required to have $R \equiv \sqrt{\Delta \eta^{2}+\Delta \phi^{2}} \leq 0.4$ and $E_{T}($ jet $)>25 \mathrm{GeV}$.

- Leptons are considered isolated if they have $p_{T}(l)>5 \mathrm{GeV}$ with visible activity within a cone of $\Delta R<0.2$ of $\Sigma E_{T}^{\text {cells }}<5 \mathrm{GeV}$.

Jets are tagged as $b$-jets if they contain a B hadron with $E_{T}(B)>15 \mathrm{GeV}, \eta(B)<3$ and $\Delta R(B$, jet $)<0.5$. We assume a tagging efficiency of $60 \%$ and light quark and gluon jets can be mis-tagged as a $b$-jet with a probability $1 / 150$ for $E_{T} \leq 100 \mathrm{GeV}, 1 / 50$ for $E_{T} \geq$ $250 \mathrm{GeV}$, with a linear interpolation for $100 \mathrm{GeV} \leq E_{T} \leq 250 \mathrm{GeV}$ (see R. Kadala et al. in ref. [85-88]).

\section{Early searches without $E_{T}^{\text {miss }}$ or electron ID}

During the early stages of data taking at the LHC with integrated luminosity of order $0.1 \mathrm{fb}^{-1}$, it is possible that $E_{T}^{\text {miss }}$ and electron identification will not be reliable observables (see refs. [80-83]). Therefore we separate our analysis into two stages:

- early searches, where no $E_{T}^{\text {miss }}$ cuts are applied and only muons are considered for the leptonic channels, and

- full analysis, using a minimum $E_{T}^{\text {miss }}$ cut and including both $e$ 's and $\mu$ 's.

In both cases we assume that the $b$-jets can be reliably tagged due to their displaced vertices as reconstructed in the micro-vertex detector or via a non-isolated muon tag. For initial searches we apply the following set of minimal cuts, labelled $\mathrm{C} 0$ : 


\begin{tabular}{|c|c|c|c|}
\hline \multicolumn{4}{|c|}{ Results after C0-based selection } \\
\hline & $\sigma(n(b) \geq 3)$ & $\sigma(n(b) \geq 4)$ & $\sigma(\mathrm{OS})$ \\
\hline $\mathrm{HSb}$ & $899 \mathrm{fb}$ & $176 \mathrm{fb}$ & $99 \mathrm{fb}$ \\
\hline $\mathrm{DR} 3 \mathrm{~b}$ & $1334 \mathrm{fb}$ & $243 \mathrm{fb}$ & $22 \mathrm{fb}$ \\
\hline $\mathrm{BG}$ & $1911 \mathrm{fb}$ & $70 \mathrm{fb}$ & $11 \mathrm{fb}$ \\
\hline
\end{tabular}

Table 3. Cross-sections for the $n(b) \geq 3,4$ and OS channels after the $\mathrm{C} 0$ cuts for the points HSb, DR3b and the background.

\section{C0 cuts:}

- Jet cuts: $n(j e t s) \geq 4$ with $E_{T}(j) \geq 50 \mathrm{GeV}, \eta(j) \leq 3$ and for the hardest jet $E_{T}(j 1) \geq$ $100 \mathrm{GeV}$,

- Lepton cuts: $E_{T}(\ell) \geq 10 \mathrm{GeV}$ and $\eta(\ell) \leq 2$,

- $S_{T} \geq 0.2$

- $n(b) \geq 1$,

where $S_{T}$ is the transverse sphericity and for now $\ell=\mu$ only.

\subsection{Multi $b$-jet signal}

As discussed in section 2 the points $\mathrm{HSb}$ and DR3b are expected to be rich in $b$-jets and possibly isolated leptons. In figure 7 we plot the $b$-jet multiplicity for the signal and backgrounds (BG) after applying the $\mathrm{C} 0$ set of cuts. As expected, the $\mathrm{BG}$ distribution falls much faster than the signal. Signal exceeds BG for $n(b) \geq 4$, where the BG is dominated by multi $b$ production $(b \bar{b}$ and $b \bar{b} b \bar{b})$. We also note that the DR3b case has larger rates for 1 $\leq n(b) \leq 4$ when compared with the HSb point, due to a lighter gluino. However, the HSb benchmark gives a larger signal for $n(b) \geq 5$, since in this case $\widetilde{Z}_{2} \rightarrow \widetilde{Z}_{1} b \bar{b}$ also contributes, and $\widetilde{Z}_{2}$ are produced at large rates from gluino cascade decay.

We note here that a signal rate above an expected SM BG level may not be sufficient to claim a discovery, due to both theoretical and experimental uncertainties in the multi- $b$ background rate. However, the overall shape of the $n(b)$ distribution should be to some extent self-normalizing, as one can fix the BG levels in the $n(b)=0,1,2$ channels, and look for a harder $n(b)$ distribution in the signal case. Thus, some of the uncertainty is removed when one looks for an excess in ratios such as $\sigma(n(b)=3) / \sigma(n(b)=1)$. Table 3 shows the $n(b) \geq 3$ and 4 cross-sections for both points and the background. The signal in the $n(b) \geq 4$ channel is at the $200 \mathrm{fb}$ level and is well above SM background levels. Such a signal may be visible with very low integrated luminosity values $\sim 0.05 \mathrm{fb}^{-1}$ !

As mentioned above, a mere excess in one or more of the multi $b$-jet channels may not be sufficient to claim discovery, due to large uncertainties in the normalization of the high jet multiplicities BGs, such as $b \bar{b}, t \bar{t}$ and $Z+2$ jets. With this in mind, we present some signal distributions with distinct shapes from the BG ones, which could help corroborate a discovery and provide some information on the sparticle masses. As discussed in section 2, 


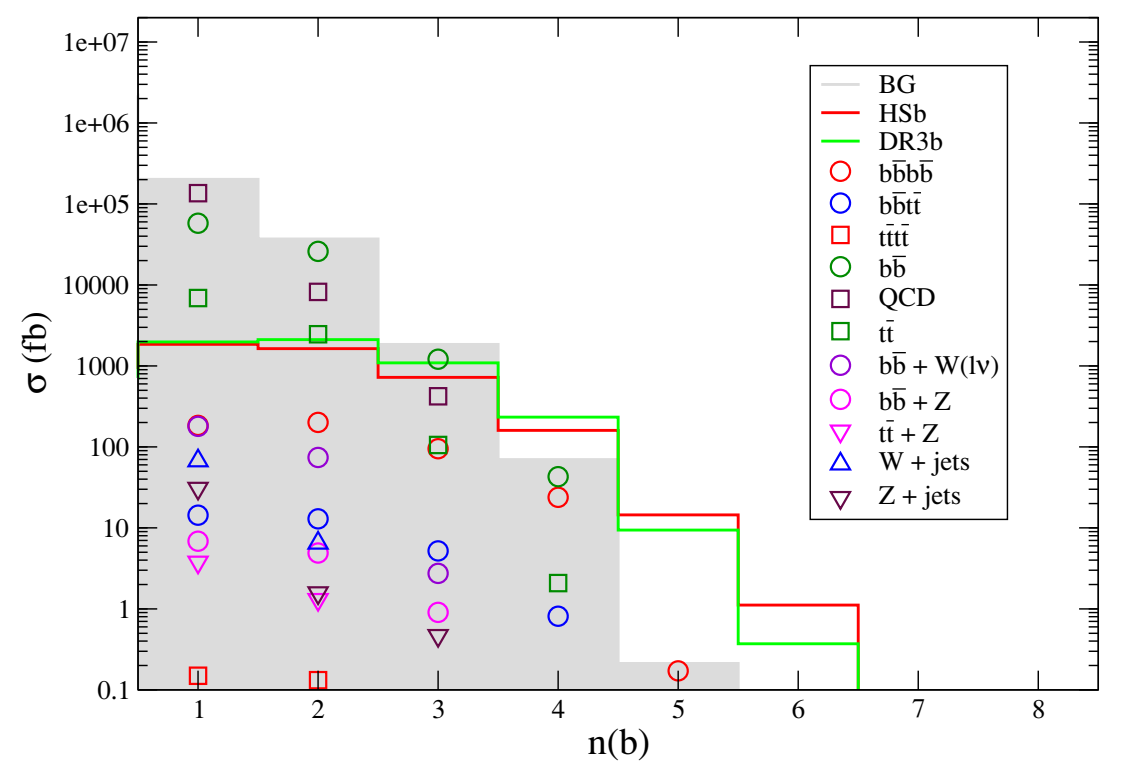

Figure 7. $b$-jet distribution after $\mathrm{C} 0$ cuts at the LHC, with $\sqrt{s}=7 \mathrm{TeV}$. We show the signal levels for the HSb (red) and DR3b (green) points along with various SM backgrounds.

events with $n(b) \geq 4$ usually come from $\tilde{g} \rightarrow \widetilde{Z}_{i} b \bar{b}$ decays. Therefore the invariant mass of the $b \bar{b}$ pair is expected to have edges at $m_{\tilde{g}}-m_{\widetilde{Z}_{i}}$. This is shown in figures 8 and 9 , where $\max \left[m_{b_{1} \bar{b}_{1}}, m_{b_{2} \bar{b}_{2}}\right]$ is plotted $^{8}$ at the parton level (dashed black line), using the correct pairing of $b$-jets, and using the parton level $b$-quark four-momentum (no hadronization or smearing). This provides an optimal distribution, useful for comparison to the more realistic case described below. As expected, in the DR3b case the $m_{\tilde{g}}-m_{\widetilde{Z}_{1}}$ mass edge is much more evident than the $m_{\tilde{g}}-m_{\widetilde{Z}_{2}}$ edge, due to the large $\tilde{g} \rightarrow \widetilde{Z}_{1}+b \bar{b}$ branching fraction, while the opposite happens for the HSb point.

At the detector level, the main difficulty in obtaining $\max \left[m_{b_{1} \bar{b}_{1}}, m_{b_{2} \bar{b}_{2}}\right]$ comes from combining the correct $b$-jets into pairs coming from the same gluino. As pointed out in ref. [74], usually the two hardest $b$-jets come from different gluinos. Furthermore, in most cases the pair coming from the same $\tilde{g}$ has smaller separation angles. Using these two facts, we label the four $b$-jets as $b_{1}-b_{4}$ ordered from highest to lowest $p_{T}$. The first and second jets will be in different pairs, so the possible combinations are: case I: $\left(b_{1}, b_{3}\right)$ and $\left(b_{2}, b_{4}\right)$ or case II: $\left(b_{1}, b_{4}\right)$ and $\left(b_{2}, b_{3}\right)$. For each case, we select the $b$-jet pair with smaller invariant mass (e.g. could be $\left(b_{2}, b_{4}\right)$ in case I and $\left(b_{1}, b_{4}\right)$ in case II, for example) and compute the $\Delta \phi$ separation for this pair. The case which gives the smaller $\Delta \phi$ value is the one selected as the "correct" pairing. After selecting the pairing, we apply a cut $\Delta R<1$ (where $R=\sqrt{\Delta \phi^{2}+\Delta \eta^{2}}$ ) on the pair with smaller invariant mass. This procedure allows for the pair with higher invariant mass to have large separation angles, and avoids cutting events near the mass edges. This pairing algorithm produces similar results to other choices (using other grouping criteria) within the statistical uncertainties of $1 \mathrm{fb}^{-1}$ of data. Using this procedure and adding the $\mathrm{C} 0$ set of cuts we obtain the solid curves shown in figures 8 and 9 ,

\footnotetext{
${ }^{8}$ Here the index $i$ in $b_{i}$ labels $b$ 's coming from the same gluino.
} 


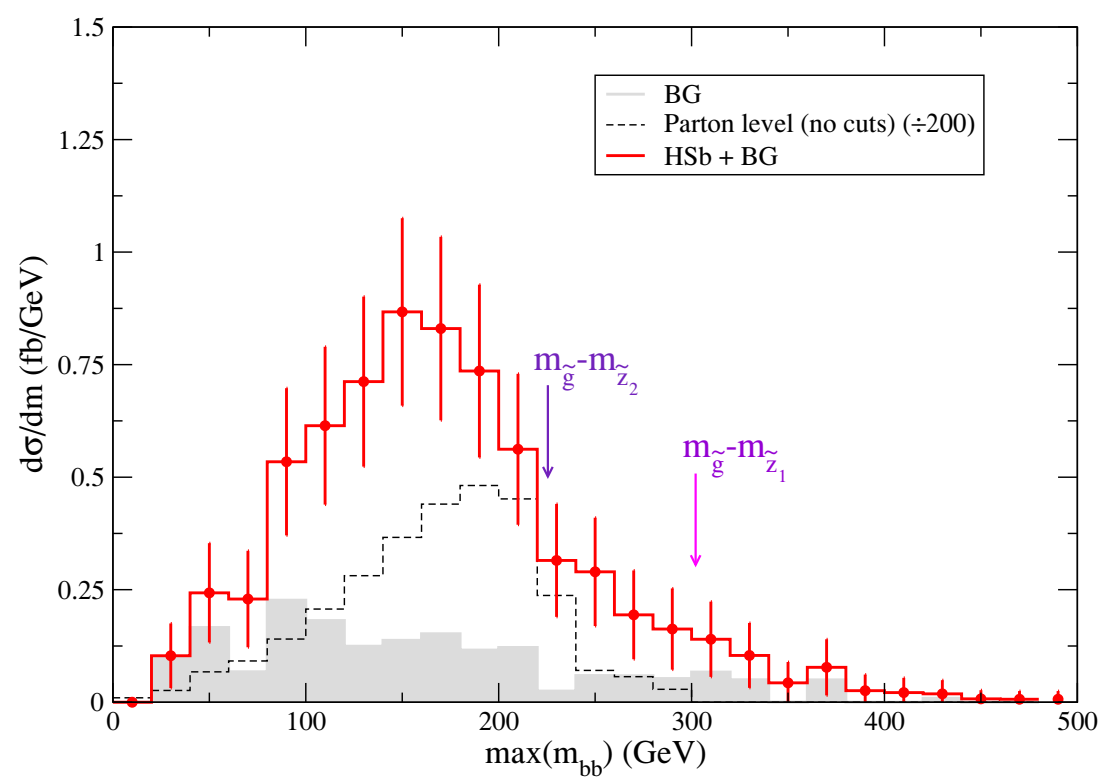

Figure 8. Maximum invariant $b b$ mass at parton level for the HSb point without cuts (black/dashed) and at detector level for the HSb plus background events (red/solid) with $n(b) \geq$ $4, \Delta R\left(b_{2}, \bar{b}_{2}\right)<1$ after the $\mathrm{C} 0$ cuts (see text). The BG distribution (gray) and the statistical error bars for $1 \mathrm{fb}^{-1}$ of integrated luminosity are also shown.

where the BG contribution was added to the signal. We also show the statistical error bars for $1 \mathrm{fb}^{-1}$ of integrated luminosity. As can be seen, the invariant-mass distributions have the expected shape, although the mass edges seem to require higher integrated luminosity to become statistically relevant. Once the mass edges are established, and a knowledge of $m_{\widetilde{Z}_{2}}$ and $m_{\widetilde{Z}_{1}}$ is gained or assumed (say, from gaugino mass unification), then some knowledge of $m_{\tilde{g}}$ may be extracted.

\subsection{Dimuon channels}

In figure 10 we show the muon multiplicity for the HSb and DR3b signal points and the BG. For $n(\mu)=0,1$, the BG is well above the signal, but for $n(\mu) \geq 2$, signal starts to dominate over the BG. As expected from the discussion in section 2, the DR3b has much smaller rates to multileptons. Separating the $n(\mu)=2$ channel into opposite sign (OS) and same sign (SS) muons, we see that almost all the signal comes from the OS dimuon case. Due to the small $\tilde{g} \rightarrow \widetilde{W}_{1}^{-} t \bar{b}+$ c.c. branching fraction, the SS signal is almost 2 orders of magnitude below the OS one, which makes it irrelevant for luminosities $\lesssim 1 \mathrm{fb}^{-1}$. As seen in table 3 , the OS cross-section for the HSb point is around $100 \mathrm{fb}$ and has a discovery potential similar to the multi $b$-jet channel, while the DR3b benchmark will require more integrated luminosity to be seen in the OS dimuon channel.

As is well known, the invariant mass of OS muons has a mass edge at $m_{\widetilde{Z}_{2}}-m_{\widetilde{Z}_{1}}$, since most OS muons come from $\widetilde{Z}_{2} \rightarrow \widetilde{Z}_{1} \mu^{+} \mu^{-}$decays. In figure 11 , we show the $m\left(\mu^{+} \mu^{-}\right)$ distribution for the HSb and DR3b points. As discussed in section 2, the DR3b point has small leptonic rates but may still be visible above background. In both cases, the mass 


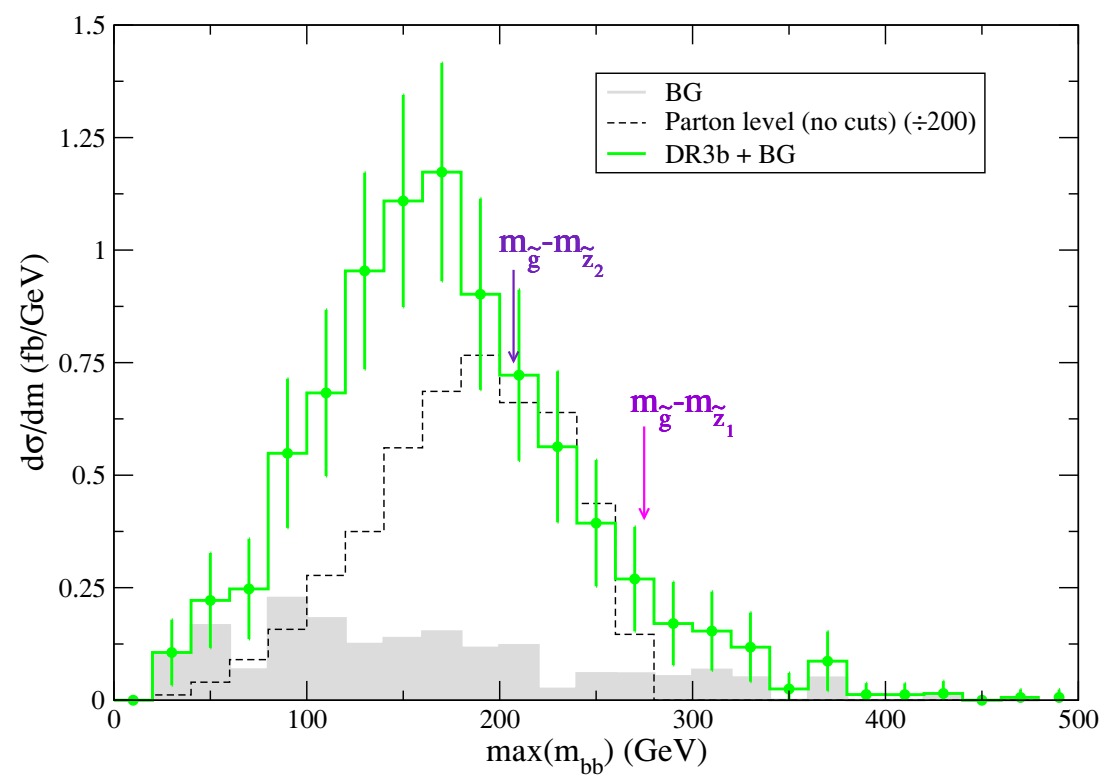

Figure 9. Maximum invariant $b b$ mass at parton level for the DR3b point without cuts (black/dashed) and at detector level for the DR3b plus background events (green/solid) with $n(b) \geq$ $4, \Delta R\left(b_{2}, \bar{b}_{2}\right)<1$ after the $\mathrm{C} 0$ cuts (see text). The BG distribution (gray) and the statistical error bars for $1 \mathrm{fb}^{-1}$ of integrated luminosity are also shown.

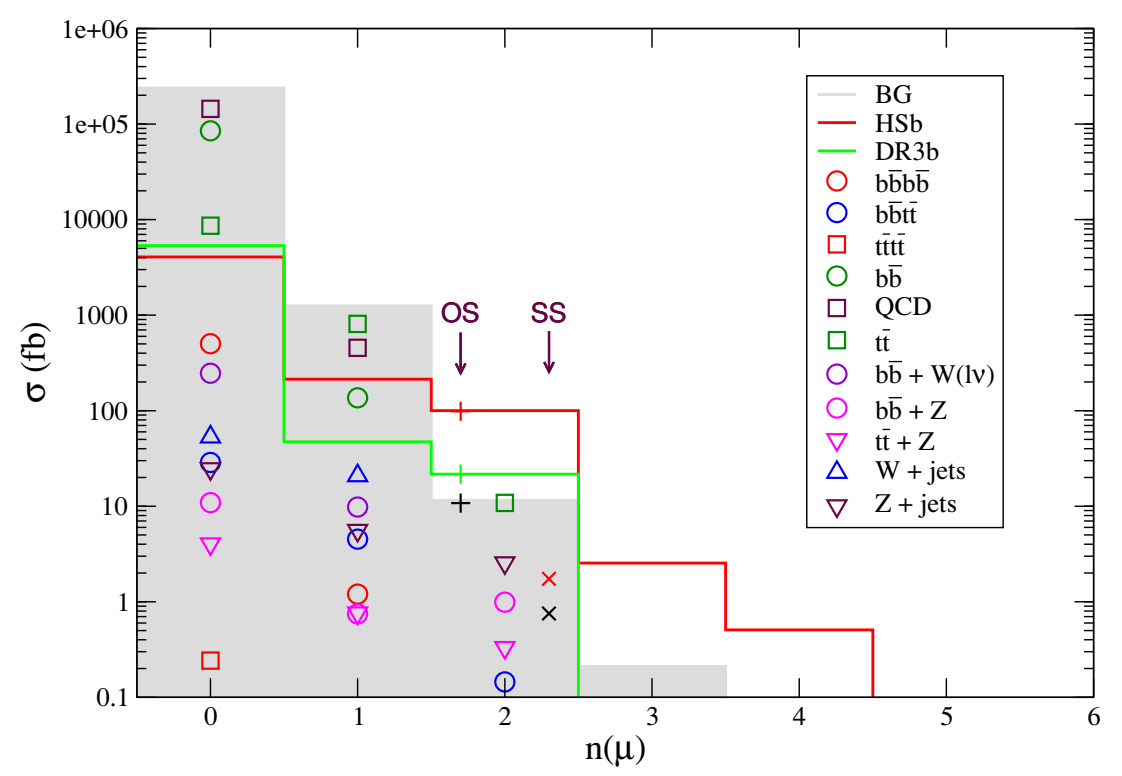

Figure 10. Muon distribution after $\mathrm{C} 0$ cuts at the LHC, with $\sqrt{s}=7 \mathrm{TeV}$. We show the signal levels for the HSb (red) and DR3b (green) points along with various SM backgrounds. In the $n(\mu)=2$ bin, the left (pluses) and right (crosses) columns show the background (black) and signal components for OS (with invariant mass cuts) and SS dimuons.

edges are visible and give the correct $m_{\widetilde{Z}_{2}}-m_{\widetilde{Z}_{1}}$ values. We also show the statistical error bars for the combined signal plus background for $1 \mathrm{fb}^{-1}$ of integrated luminosity. From 


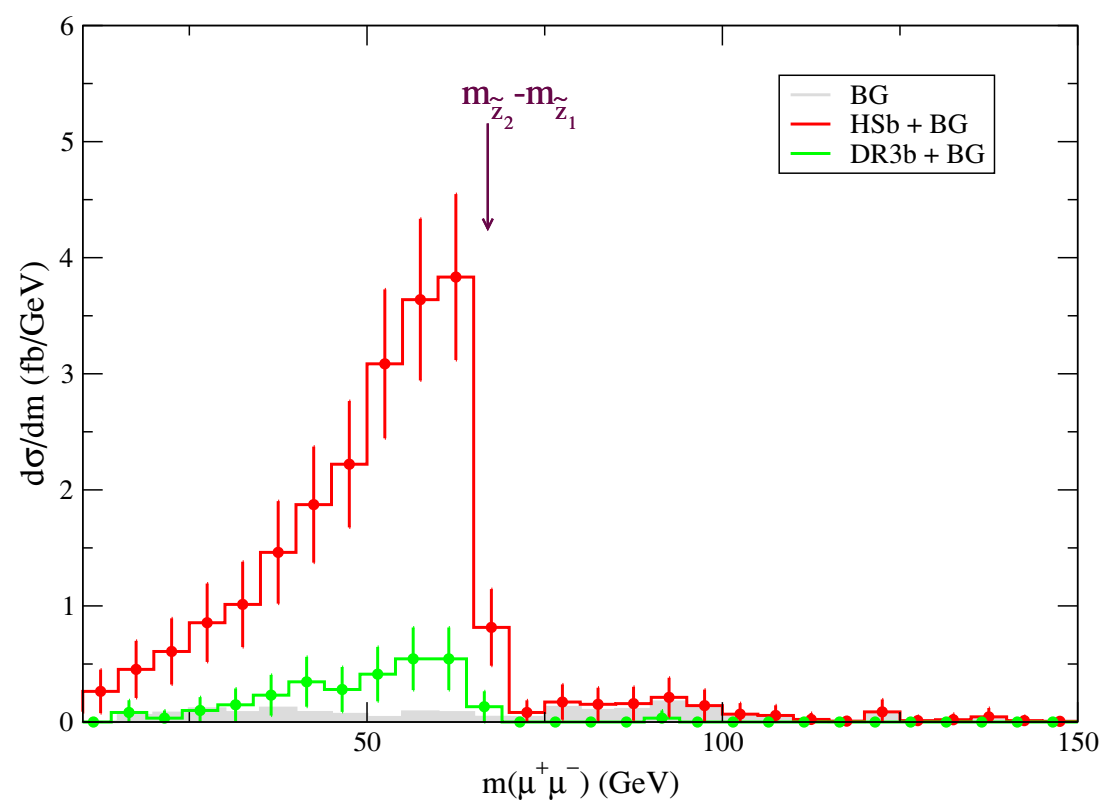

Figure 11. OS dimuon invariant mass for the HSb (red) and DR3b (green) points plus background events after the $\mathrm{C} 0$ cuts (see text). The BG distribution (gray) and the statistical error bars for $1 \mathrm{fb}^{-1}$ of integrated luminosity are also shown.

figure 11, we can see that the HSb point gives a statistically significant edge while the DR3b case may require higher integrated luminosities.

Finally, we point out that in both the $n(b) \geq 4$ and OS dimuon channels the invariant mass distributions shown in figures 8,9 and 11 have distinct features from the BG, which should corroborate a discovery claim.

\subsection{Early SUSY search: reach results}

To estimate the LHC reach in the multi- $b$ and multi- $\mu$ channels, we plot in figure 12 the signal cross section for the HS and DR3 model lines versus $m_{\tilde{g}}$ using cuts C0 plus $a) \cdot n(b) \geq 3$ and $b) \cdot n(b) \geq 4$, along with the expected $\mathrm{BG}$ rate. We also plot the $5 \sigma$ discovery lines for integrated luminosity values 0.1 and $0.2 \mathrm{fb}^{-1}$. The significance in $\sigma \mathrm{s}$ is derived from the p-value corresponding to the number of $\mathrm{S}+\mathrm{B}$ events in a Poisson distribution with a mean that equals to the number of background events. ${ }^{9}$ For both the HS and DR3 model lines, the approximate $5 \sigma$ LHC reach extends to $m_{\tilde{g}} \sim 360 \mathrm{GeV}$ for $0.1 \mathrm{fb}^{-1}$, and $\sim 400 \mathrm{GeV}$ for $0.2 \mathrm{fb}^{-1}$. We remind the reader that this is comparable to what Tevatron experiments can achieve using $E_{T}^{\text {miss }}$ cuts and $>5 \mathrm{fb}^{-1}$ of integrated luminosity [73].

The reach using cuts $\mathrm{C} 0$ and requiring an OS dimuon pair is shown in figure 13. In this case, the reach in the HS model is similar to the multi- $b$ reach: LHC should explore to $m_{\tilde{g}} \sim 360 \mathrm{GeV}$ with $0.1 \mathrm{fb}^{-1}$, and $m_{\tilde{g}} \sim 400 \mathrm{GeV}$ with $0.2 \mathrm{fb}^{-1}$. In the DR3 model line, however, there is no reach in the OS dimuon channel at these low values of integrated

\footnotetext{
${ }^{9}$ H.B. Prosper, private code for calculation of significance from p-values.
} 

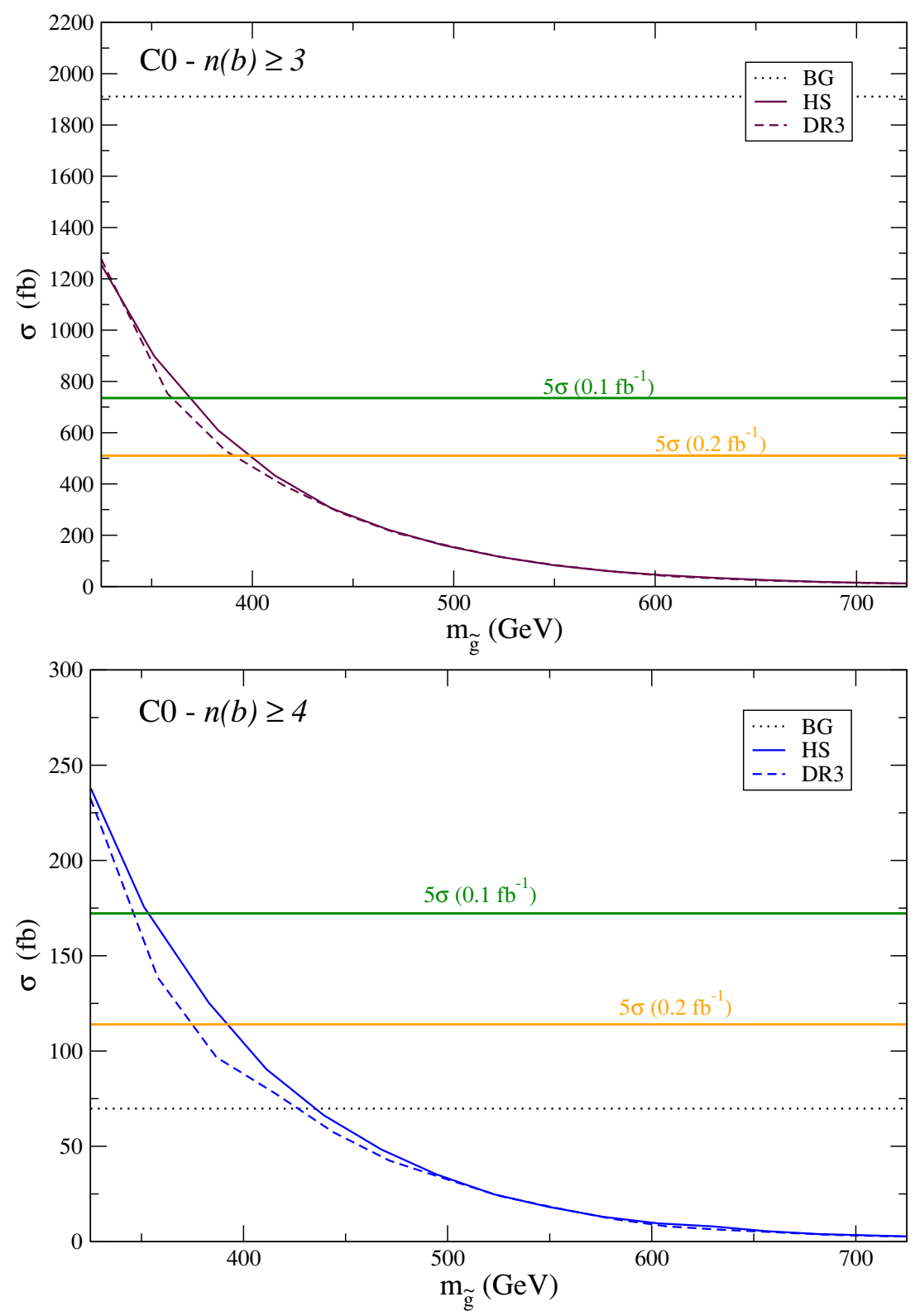

Figure 12. Early LHC reach for Yukawa-unified SUSY using cuts $C 0$ plus $n_{b} \geq 3$ and $n_{b} \geq 4$.

luminosity. In fact, the rate of multi-b-jet events compared to the rate for OS dimuon events would be one way to distinguish early-on whether one might be seeing SUSY in the HS or the DR3 model case.

We also point out that despite giving the maximum reach for both models, the $n(b) \geq 3$ channel has a small signal/BG ratio, what makes it more dependent on the knowledge of the background. 


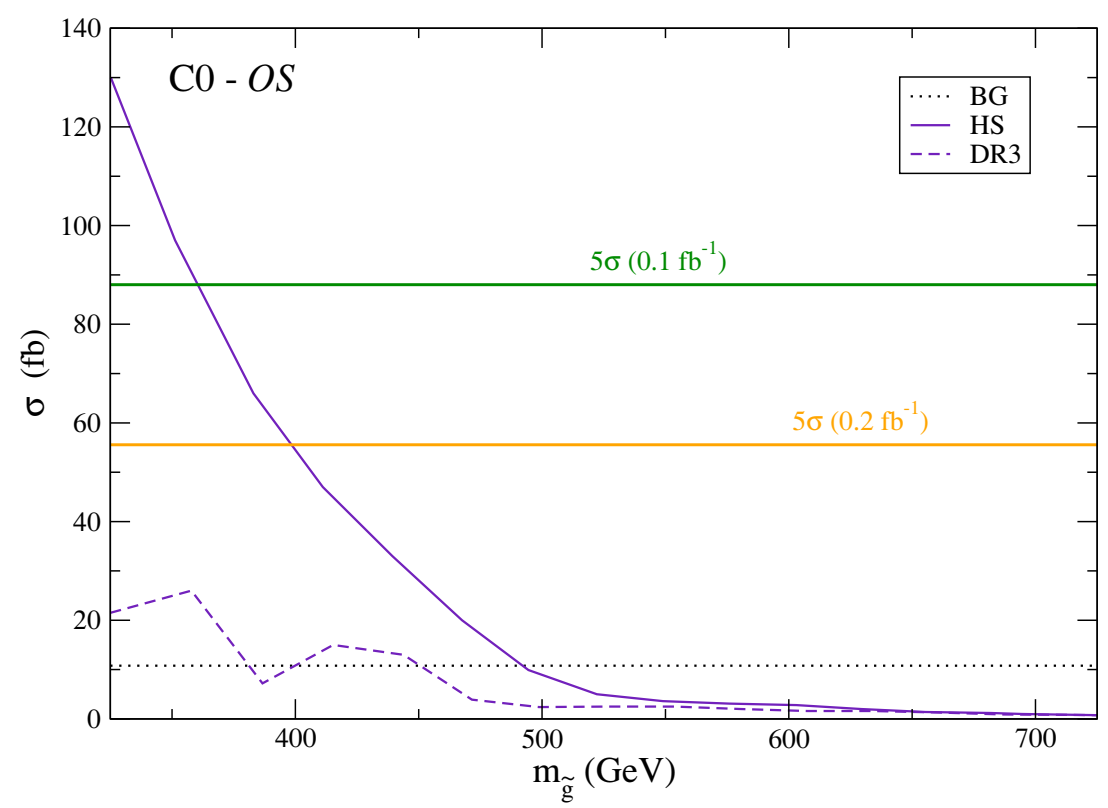

Figure 13. Early LHC reach for Yukawa-unified SUSY using cuts $C 0$ plus requiring OS dimuons.

\section{$5 \quad$ Analysis including $E_{T}^{\text {miss }}$ cut and electron ID}

As the experiments accumulate data, knowledge of the detectors and their response to SM background will improve. Also, at some point in time, the LHC center-of-mass energy will increase beyond $7 \mathrm{TeV}$ into the $10 \mathrm{TeV}$ range. To be conservative, we will continue our analysis assuming $\sqrt{s}=7 \mathrm{TeV}$. Moving to higher values of $\sqrt{s}$ should only increase the possibility of discovering new, high mass matter states.

As detector response becomes better understood, it will be possible to utilize both $E_{T}^{\text {miss }}$ and electrons in the analysis. The $E_{T}^{\text {miss }}$ variable is well known to be a powerful discriminator between SUSY and SM events, and is considered to be the "classic" signature for SUSY. In figure 14 we show the $E_{T}^{\text {miss }}$ distributions for the BG as well as the HSb and DR3b points after applying the C0 cuts. As expected, the DR3b signal has a harder $E_{T}^{\text {miss }}$ spectrum than HSb, due to its large $\tilde{g} \rightarrow \widetilde{Z}_{1} b \bar{b}$ branching ratio.

In order to reduce most of the background and still keep considerably large crosssections for the signal, we henceforth:

- include the $E_{T}^{\text {miss }}>100 \mathrm{GeV}$ cut and

- include e's into the $\mathrm{C} 0$ leptonic cuts

into our analysis. The cuts $\mathrm{C} 0$ augmented with the $E_{T}^{\text {miss }}$ cut and inclusion of $e$ 's, but with no $n(b)$ requirement, will be called $\mathrm{C} 1$ cuts:

\section{C1 cuts:}

- Jet cuts: $n(j e t s) \geq 4$ with $E_{T}(j) \geq 50 \mathrm{GeV}, \eta(j) \leq 3$ and for the hardest jet $E_{T}(j 1) \geq$ $100 \mathrm{GeV}$, 


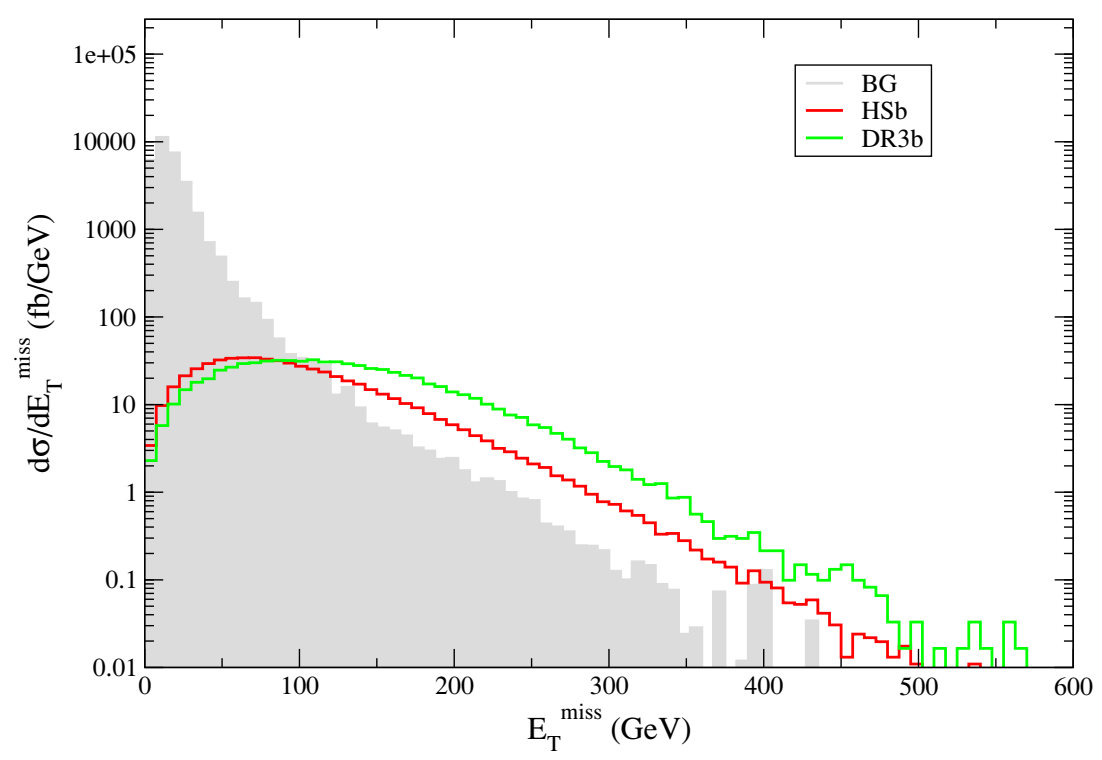

Figure 14. $E_{T}^{\text {miss }}$ distribution for the BG (gray), HSb (red) and DR3b (green) points after the C0 cuts (see text).

- Lepton cuts: $E_{T}(\ell) \geq 10 \mathrm{GeV}$ and $\eta(\ell) \leq 2$,

- $S_{T} \geq 0.2$,

- $E_{T}^{\text {miss }}>100 \mathrm{GeV}$,

where $\ell=\mu, e$.

\subsection{Multi $b$-jet $+E_{T}^{\text {miss }}$ channel}

The main effect of adding an $E_{T}^{\text {miss }}$ cut to our previous analysis is the drastic reduction of background in the multi $b$-jet channel. However, the signal will also be significantly reduced, and so will be the statistics in the invariant mass distributions.

The $n(b)$ distribution after $\mathrm{C} 1$ cuts is shown in figure 15. Now the signal's peak at $n(b)=1,2$ is visible above the BG, and the hard distribution in $n(b)$ should be a striking signature for both the DR3b and HSb models, since the combined signal plus BG distribution becomes approximately flat for $0 \leq n(b) \leq 2$. The $E_{T}^{\text {miss }}$ cut reduces most of the $b \bar{b}$ and $b \bar{b} b \bar{b}$ backgrounds, leaving $t \bar{t}$ as the dominant one for $n(b) \geq 1$. This results in a considerable reduction of the $\mathrm{BG}$ in the $n(b)=1,2$ and 3 bins, where now the signal/background ratio is larger than one. The cross-sections for $n(b) \geq 3,4$ are shown in table 4 . Due to the large signal cross-section in these bins, the signal could be visible with less than $0.05 \mathrm{fb}^{-1}$ of integrated luminosity (in the happy case where an $E_{T}^{\text {miss }}$ measurement is immediately viable)!

The invariant mass distributions from figures 8 and 9 are now re-plotted in figures 16 and 17 after the $E_{T}^{\text {miss }}$ cut is included. Now, due to the drastic reduction in the background, the $m_{b \bar{b}}$ distribution is nearly free of BG events. However, the decrease in the signal 


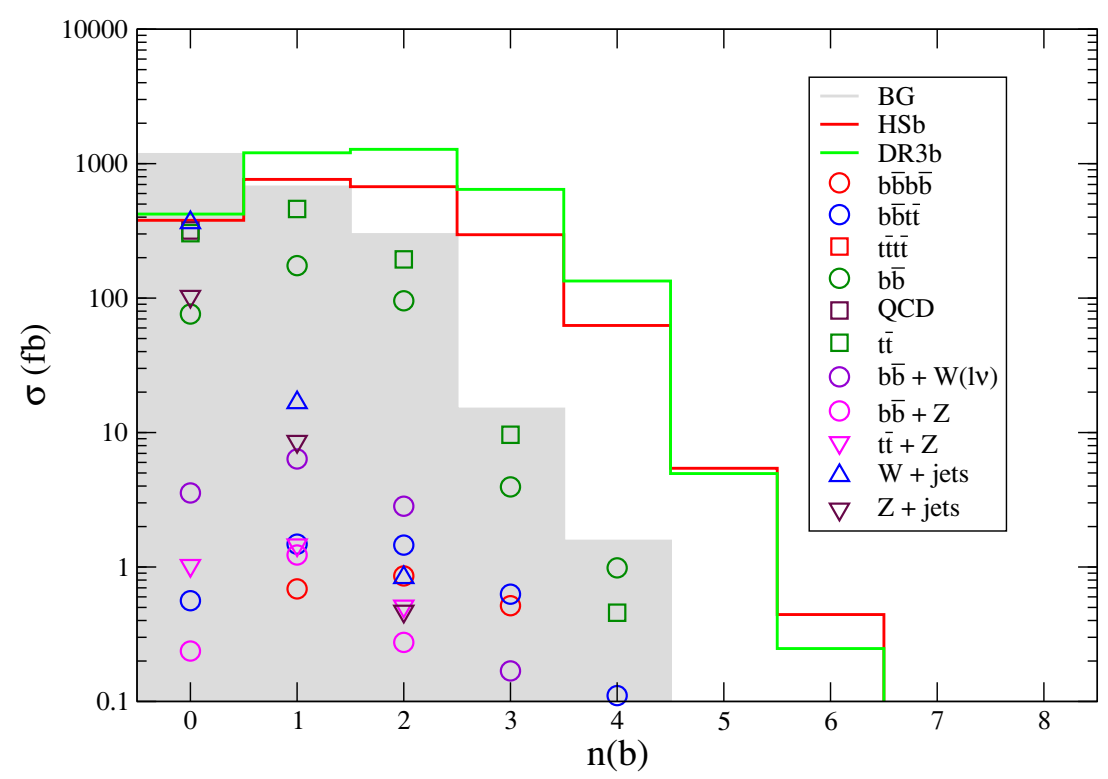

Figure 15. $b$-jet distribution after $\mathrm{C} 1$ cuts at the LHC, with $\sqrt{s}=7 \mathrm{TeV}$. We show the signal levels for the HSb (red) and DR3b (green) points along with various SM backgrounds.

\begin{tabular}{|c|c|c|c|}
\hline \multicolumn{4}{|c|}{ Results after C1-based selection } \\
\hline & $\sigma(n(b) \geq 3)$ & $\sigma(n(b) \geq 4)$ & $\sigma(\mathrm{OS})$ \\
\hline $\mathrm{HSb}$ & $364 \mathrm{fb}$ & $68 \mathrm{fb}$ & $81 \mathrm{fb}$ \\
\hline $\mathrm{DR} 3 \mathrm{~b}$ & $782 \mathrm{fb}$ & $139 \mathrm{fb}$ & $23 \mathrm{fb}$ \\
\hline $\mathrm{BG}$ & $16 \mathrm{fb}$ & $2 \mathrm{fb}$ & $9 \mathrm{fb}$ \\
\hline
\end{tabular}

Table 4. Cross-sections for the $n(b) \geq 3,4$ and OS channels after the $\mathrm{C} 1$ cuts for the points HSb, DR3b and the background.

statistics makes it impossible to obtain any information on the sparticle masses from the shape of $\max \left[m_{b_{1} \bar{b}_{1}}, m_{b_{2} \bar{b}_{2}}\right]$.

A quantity that may still reveal some information on the gluino mass scale is the effective mass, $M_{\mathrm{eff}}=\sum p_{T}(j e t s)+E_{T}^{\text {miss }}$, plotted in figure 18 for the HSb and DRb points, as well as for two points on the HS and DR3 model lines with heavier gluinos $\left(m_{\tilde{g}}=576 \mathrm{GeV}\right.$ and $m_{\tilde{g}}=581 \mathrm{GeV}$, respectively). From figure 18 we see that the effective mass distribution peaks at higher values for heavier gluinos, but the correlation seems to be too weak to provide any precise measurement of the gluino mass.

\subsection{Multi-lepton channels}

In figure 19, we show the isolated lepton multiplicity distribution after cuts $\mathrm{C} 1$. In the $n(\ell)=0$ channel, the DR3b signal exceeds BG, while in the $n(\ell)=2$ channel, the HSb signal exceeds BG. In the $n(\ell)=1$ channel, BG from $t \bar{t}$ production is larger than both signal cases. One can pull out a better signal rate in the $1 \ell$ channel by imposing in addition a $M_{T}\left(\ell, E_{T}^{\text {miss }}\right)>100 \mathrm{GeV}$ cut, as is well known [96]. 


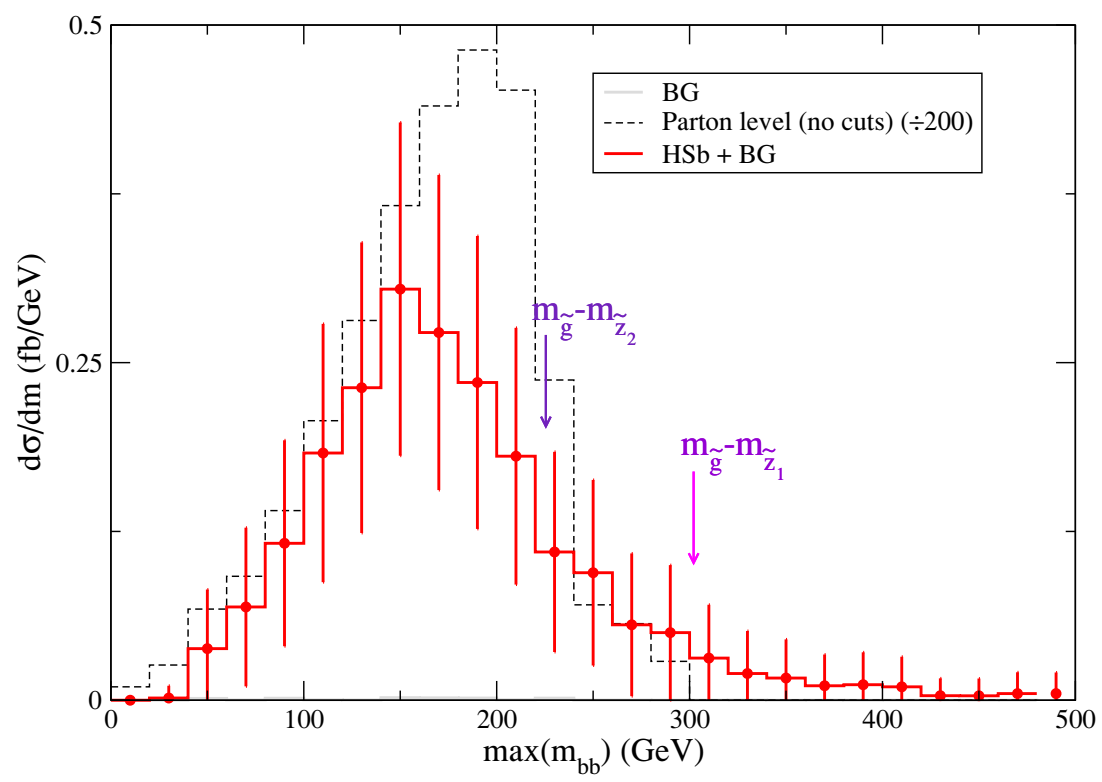

Figure 16. Same as figure 8 , but including the $E_{T}^{\text {miss }}>100 \mathrm{GeV}$ cut.

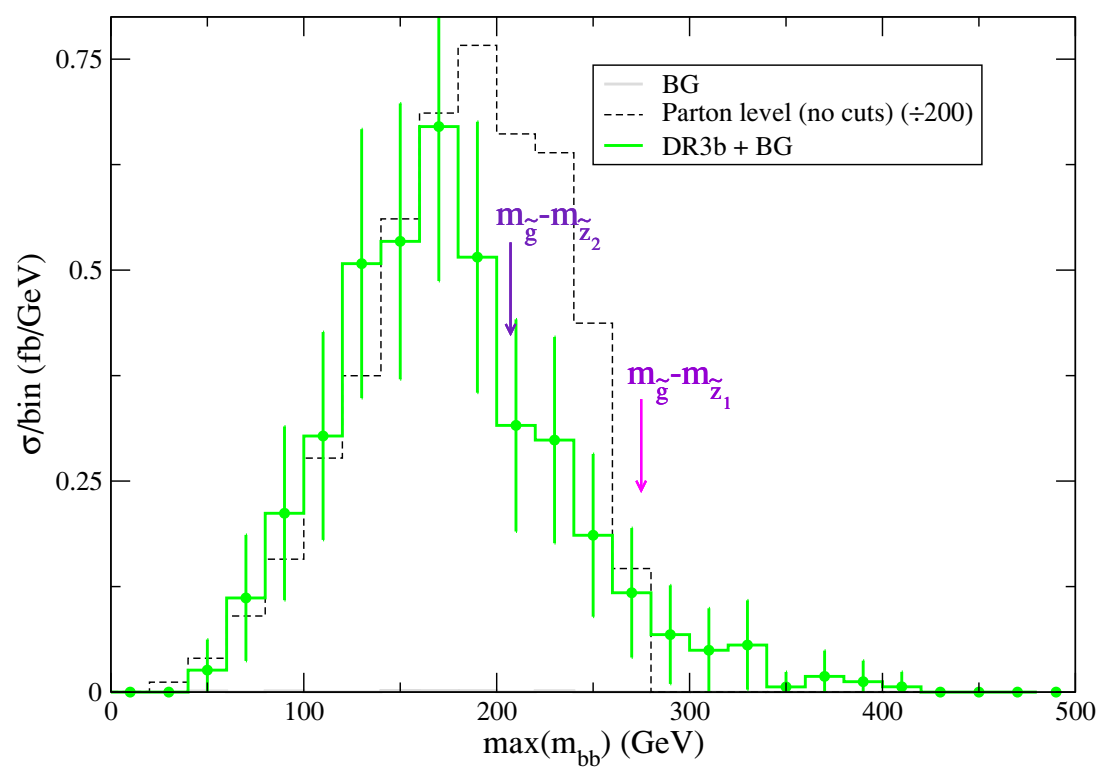

Figure 17. Same as figure 9 , but including the $E_{T}^{\text {miss }}>100 \mathrm{GeV}$ cut.

We divide the $n(\ell)=2$ channel into opposite-sign/same-flavor (OS/SF) events $\left(e^{+} e^{-}\right.$ or $\left.\mu^{+} \mu^{-}\right)$, and same-sign events. For the OS/SF channel, we see the $E_{T}^{\text {miss }}$ cut has little effect on the signal-to-BG ratio, since the background, mainly $t \bar{t}$ production, also has large $E_{T}^{\text {miss }}$. Again, we expect the entire distribution to be self normalizing, since $t \bar{t}$ is the dominant $\mathrm{BG}$, and one can fix the total $t \bar{t}$ cross section by normalizing to the $1 \ell$ channel. Then the $n(\ell)$ distribution should be harder than expected from just SM physics if a SUSY signal is present. 


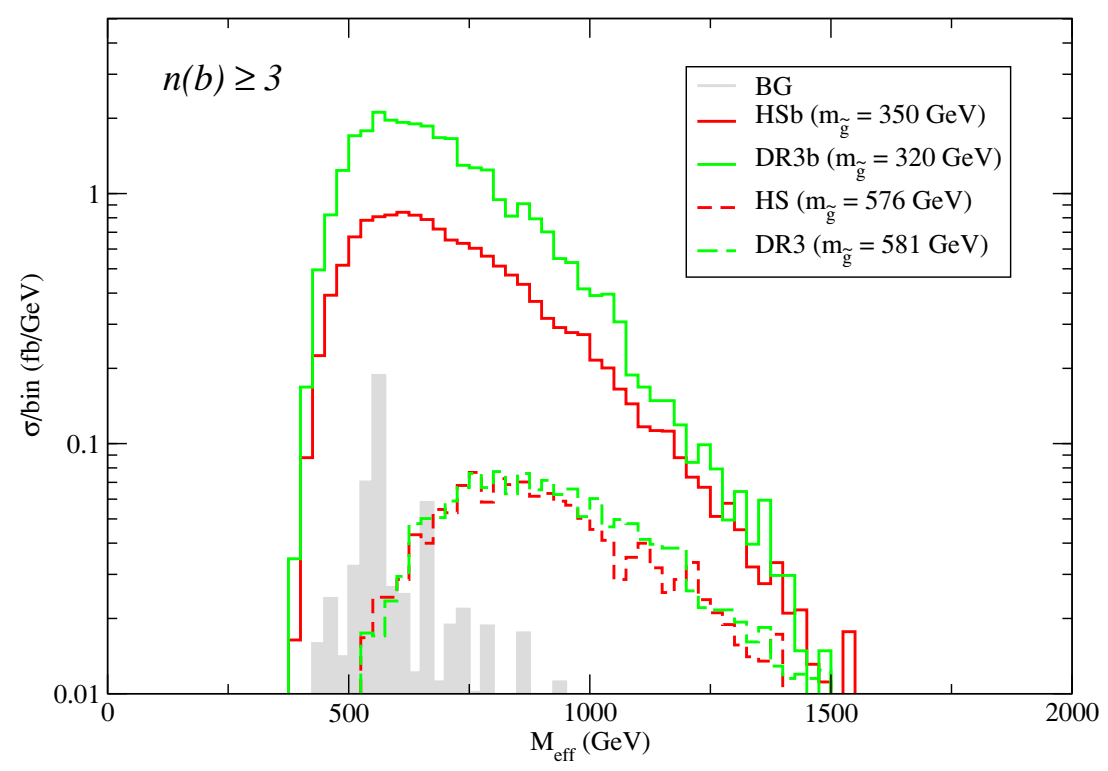

Figure 18. Effective mass scale of events with $n(b) \geq 3$ after $\mathrm{C} 1$ cuts for the BG, points HSb and $\mathrm{DRb}$, and two points on the HS and DR3 model lines with heavier gluinos $\left(m_{\tilde{g}}=576 \mathrm{GeV}\right.$ and $m_{\tilde{g}}=581 \mathrm{GeV}$, respectively).

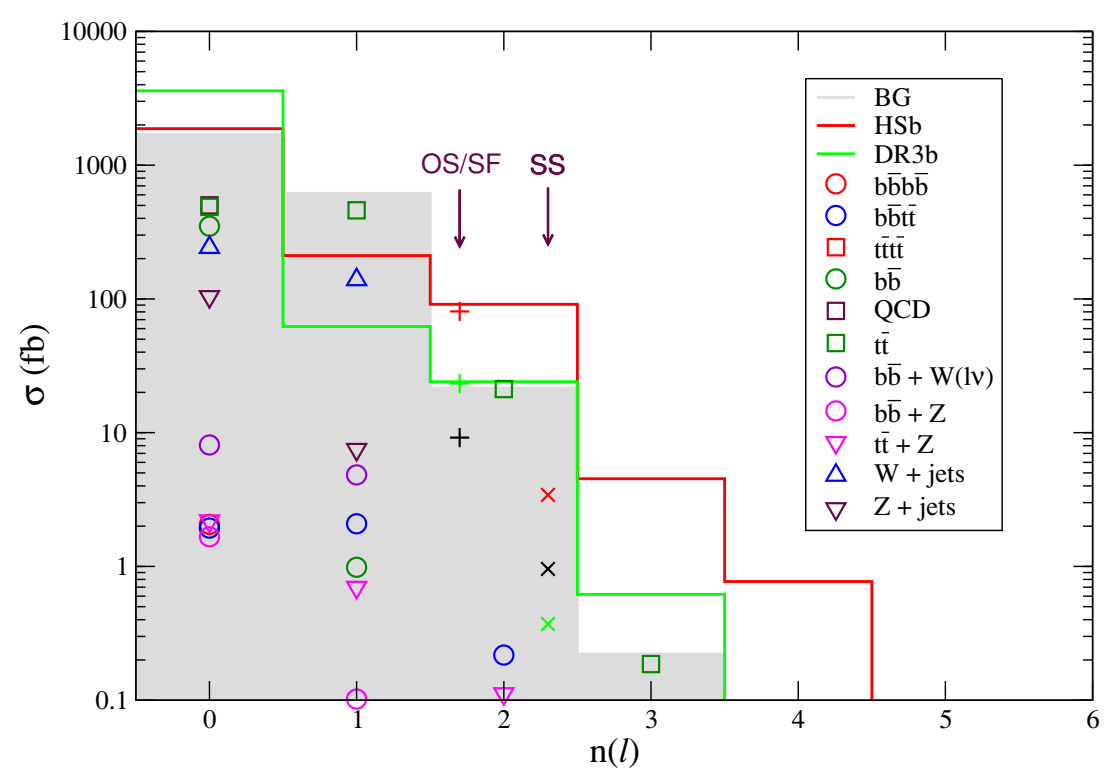

Figure 19. Lepton multiplicity distribution after $\mathrm{C} 1$ cuts at the LHC, with $\sqrt{s}=7 \mathrm{TeV}$. We show the signal levels for the HSb (red) and DR3b (green) points along with various SM backgrounds. In the $n(\ell)=2$ bin, the left (pluses) and right (crosses) columns show the background (black) and signal components for OS/SF and SS dileptons.

In figure 20, we show the OS/SF dilepton invariant mass distribution after cuts $\mathrm{C} 1$. Now, despite the negligible background, the reduction in the signal makes the mass edges less visible. In particular, the dilepton invariant mass still shows the $m_{\widetilde{Z}_{2}}-m_{\widetilde{Z}_{1}}$ edge, but with a smaller statistical significance as evident from the error bars. Performing a 


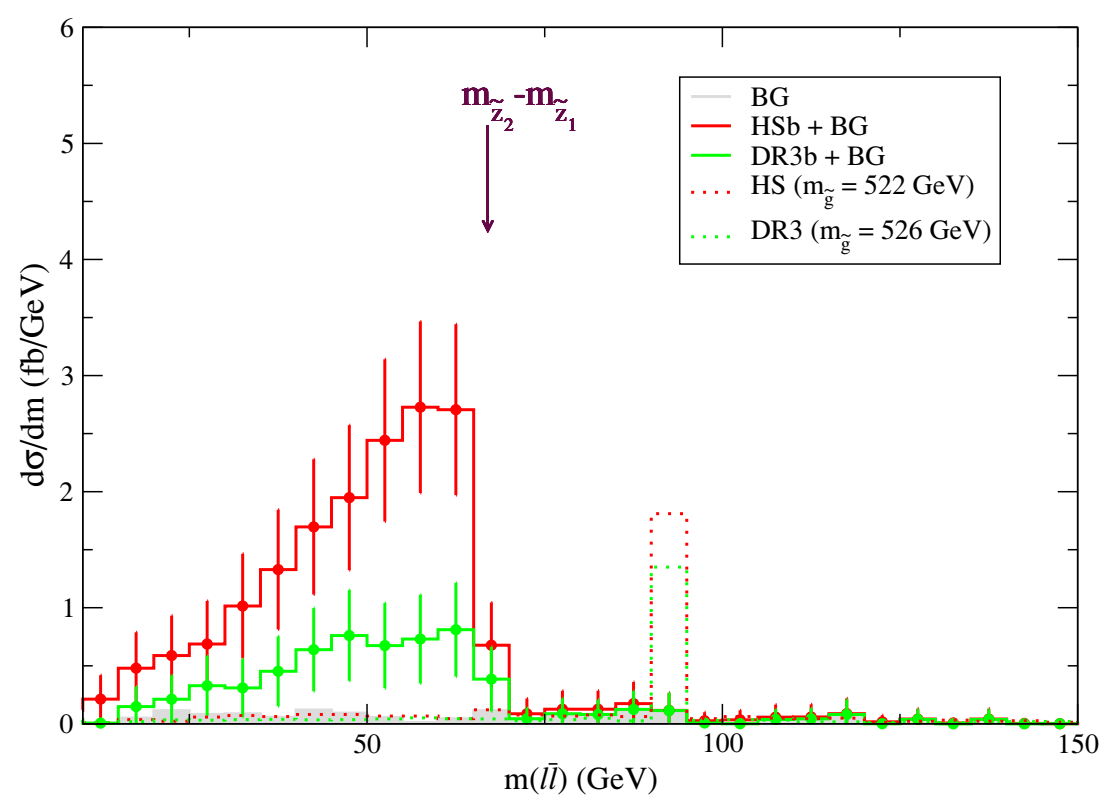

Figure 20. OS dilepton ( $\mu$ 's and $e$ 's) invariant mass for the HSb (red) and DR3b (green) points plus background events after the $\mathrm{C} 1$ cuts (see text). The BG distribution (gray) and the statistical error bars for $1 \mathrm{fb}^{-1}$ of integrated luminosity are also shown.

different-flavor subtraction may reduce the already negligible BG even further.

\subsection{Jets plus $Z \rightarrow \ell \bar{\ell}+E_{T}^{\text {miss }}$ signal}

We also show in figure 20 two additional HS and DR3 cases with $m_{\tilde{g}} \sim 525 \mathrm{GeV}$. In this case, according to figure 6 , the value of $m_{\widetilde{Z}_{2}}$ is high enough that the two-body decay $\widetilde{Z}_{2} \rightarrow \widetilde{Z}_{1} Z$ is now dominant. We then expect a signature of multiple $b$-jets plus $E_{T}^{\text {miss }}$ plus a dilepton pair which reconstructs to $m\left(\ell^{+} \ell^{-}\right) \simeq M_{Z}[97,98]$. While signal rates can be very high for this channel, SM background is low, coming from processes such as $t \bar{t}$ and $Z+t \bar{t}$.

\subsection{LHC reach for Yukawa-unified SUSY using $E_{T}^{\text {miss }}$ and $e$ ID}

Next, we investigate the full LHC reach for Yukawa-unified SUSY along the HS and DR3 model lines. First, we require the cut set $\mathrm{C} 1$, which includes $E_{T}^{\text {miss }}>100 \mathrm{GeV}$, and then require $n(b) \geq 2$ or $n(b) \geq 3$. The SM background level and $5 \sigma$ level for 0.2 and $1 \mathrm{fb}^{-1}$ are shown in the plots of figure 21, along with expected signal rates from the HS and DR3 model lines. For the $n(b) \geq 2$ case, we find an LHC reach for Yukawa-unifed SUSY out to $m_{\tilde{g}}=500(600) \mathrm{GeV}$ for $0.2(1) \mathrm{fb}^{-1}$. The reach is largely independent of whether one is in the HS or the DR3 model.

For $n(b) \geq 3$, the SM background is greatly reduced. In this case, we find a reach to $m_{\tilde{g}}=540(630) \mathrm{GeV}$ for $0.2(1) \mathrm{fb}^{-1}$. Again, the reach in the multi- $b$-jet channel is largely independent of the model line, since both give large numbers of $b$-jets $+E_{T}^{\text {miss }}$ in the final state. We have also calculated the reach in the $n(b) \geq 4$ channel; here, the result is qualitatively similar to that obtained in the $n(b) \geq 2$ case. 

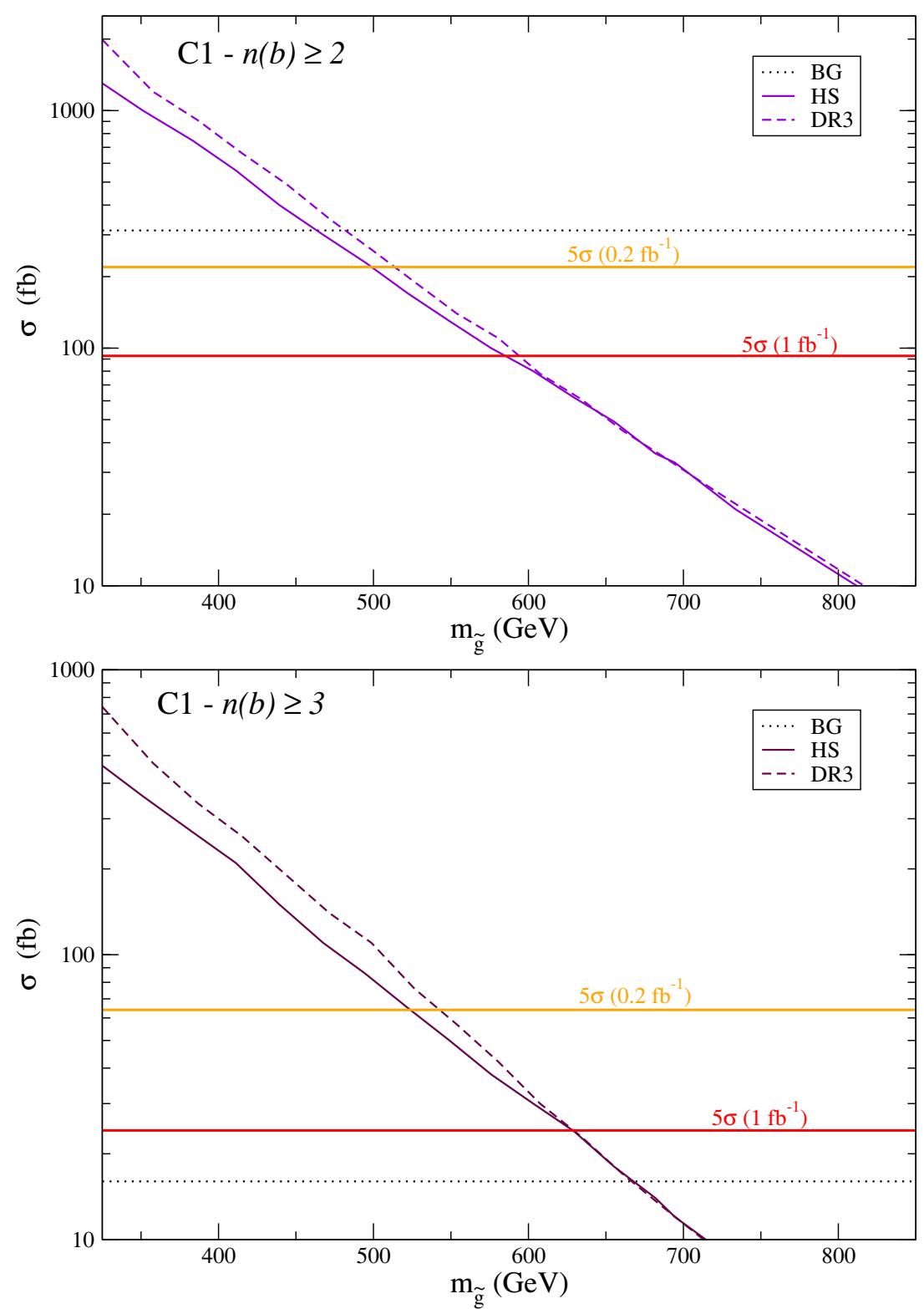

Figure 21. Early LHC reach for Yukawa-unified SUSY using cuts $C 1$ plus $n_{b} \geq 2$ and $n_{b} \geq 3$.

Next, in figure 22, we show the LHC reach for Yukawa-unified SUSY using the $C 1$ cuts plus requiring a pair of OS/SF dileptons. In this case, the LHC reach is model dependent. For the HS model, where we obtain a high rate for $\tilde{g} \rightarrow b \bar{b} \widetilde{Z}_{2}$ decays, we find a reach up to $m_{\tilde{g}}=400$ (500) $\mathrm{GeV}$ for 0.2 (1) $\mathrm{fb}^{-1}$ of integrated luminosity. For the DR3 model line, there is no reach for $0.2 \mathrm{fb}^{-1}$, since here the $\tilde{g} \rightarrow b \bar{b} \widetilde{Z}_{1}$ decay is dominant. With $1 \mathrm{fb}^{-1}$, however, a signal with $5 \sigma$ significance should be visible for $m_{\tilde{g}} \sim 300-450 \mathrm{GeV}$.

For $m_{\tilde{g}} \gtrsim 450 \mathrm{GeV}$, the two body decay mode $\widetilde{Z}_{2} \rightarrow \widetilde{Z}_{1} Z$ opens up and we expect to reconstruct $Z \rightarrow e^{+} e^{-}$and $Z \rightarrow \mu^{+} \mu^{-}$within the class of signal events. Here, we will adopt cuts $\mathrm{C} 1$, but in addition require a OS/SF dilepton pair with $75 \mathrm{GeV}<m\left(\ell^{+} \ell^{-}\right)<$ $105 \mathrm{GeV}\left(\right.$ cuts $\left.C 1^{\prime}\right)$. For this topology- $\geq 4$ jets $+E_{T}^{\text {miss }}+Z \rightarrow \ell^{+} \ell^{-}-$the dominant SM 


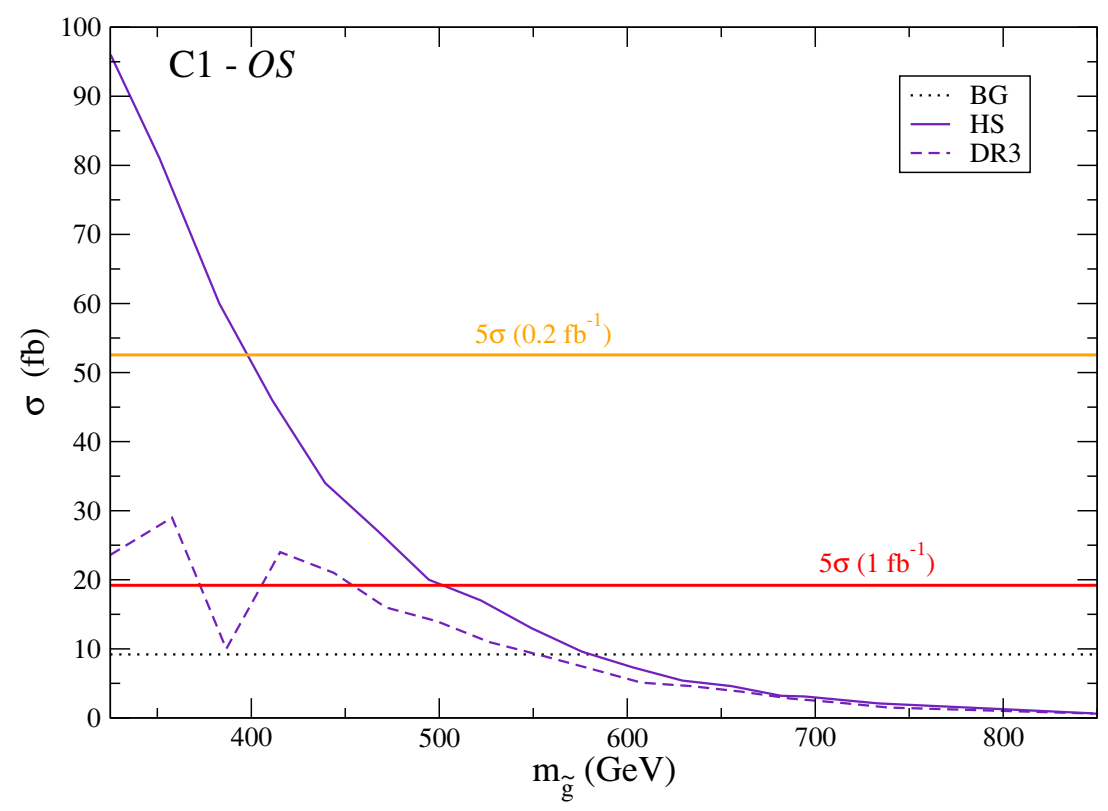

Figure 22. Early LHC reach for Yukawa-unified SUSY using cuts $C 1$ for events containing OS/SF dileptons.

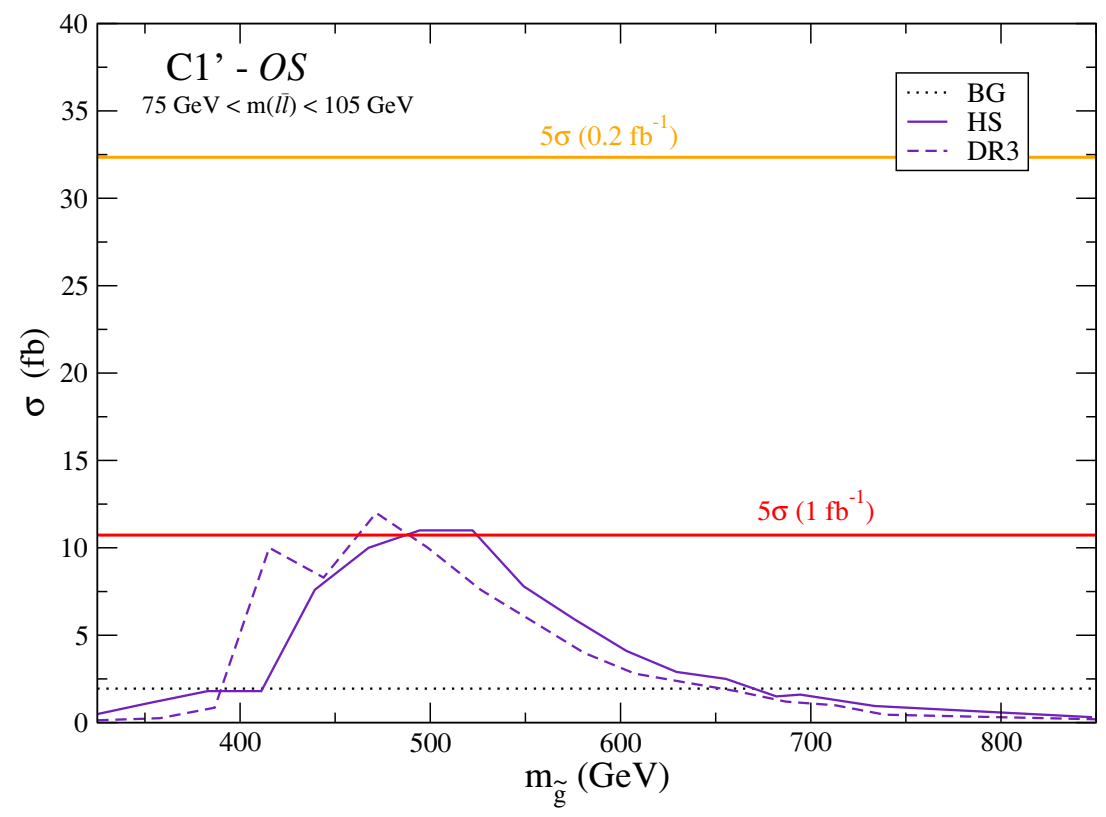

Figure 23. LHC reach for Yukawa-unified SUSY using cuts $C 1$ ' with a reconstructed leptonic $Z$ boson.

BG comes from $t \bar{t}$ production. The LHC reach is shown in figure 23. As can be seen, no significant excess is expected with $0.2 \mathrm{fb}^{-1}$ of data. However, for $\gtrsim 1 \mathrm{fb}^{-1}$, we find that this topology can produce a $5 \sigma$ signal for $m_{\tilde{g}} \sim 450-530 \mathrm{GeV}$ for both the HS and DR3 model lines. 


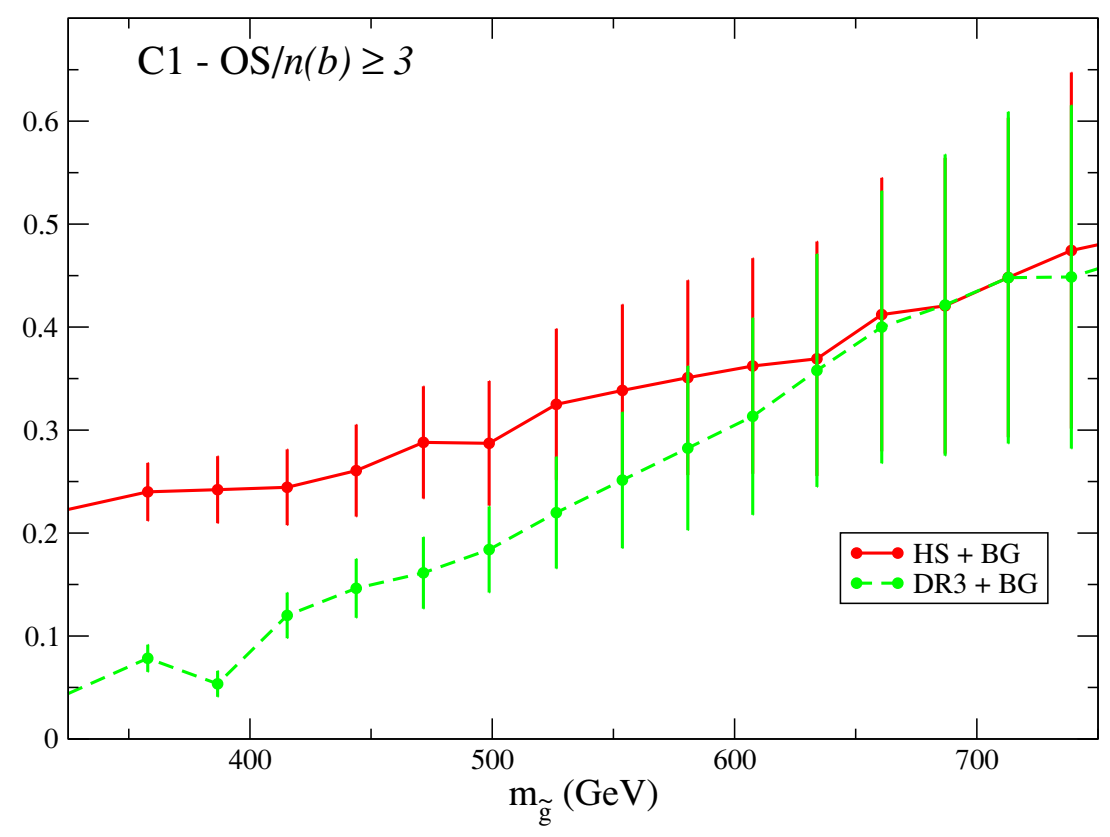

Figure 24. The ratio of the OS and $n(b) \geq 3$ cross-sections after C1 cuts for the HS and DR3 model lines plus background as a function of the gluino mass. We also show the statistical error bars for $1 \mathrm{fb}^{-1}$.

\subsection{Differentiating the HS and DR3 models}

Here we discuss the possibility of distinguishing the two models discussed so far using low luminosities and the channels investigated in the previous sections. As seen from last section results (figures 21 and 22) and the discussion in section 2, for low to moderate $m_{\tilde{g}}$ masses we expect the HS and DR3 models to have rather distinct signatures. The first one is rich in multi-b jets $(n(b) \geq 4,5)$ and OS/SF dileptons coming from the $\tilde{g} \rightarrow \widetilde{Z}_{2}+b \bar{b}$ followed by $\widetilde{Z}_{2} \rightarrow Z / Z^{*}+b \bar{b} / \ell^{+} \ell^{-}$decays, but has a softer $E_{T}^{\text {miss }}$ spectrum due to the two step cascade decays. On the other hand, the DR3 model is mainly dominated by $\tilde{g} \rightarrow \widetilde{Z}_{1}+b \bar{b}$, with small cross-sections in the multi-lepton channels, a moderate number of $b$-jets $(n(b) \geq 3,4)$ and a harder $E_{T}^{\text {miss }}$ spectrum. To explore these features and discuss how well we can distinguish both models with year one data, we plot in figure 24 the ratio of the OS and $n(b) \geq 3$ channels. As expected from the above discussion, we see that the ratio is larger for the HS model and suppressed in the DR3 case for $m_{\tilde{g}} \lesssim 600 \mathrm{GeV}$. As the gluino mass increases, the $\widetilde{W}_{1}+b t$ and $\widetilde{Z}_{1}+t \bar{t}$ gluino decay channels become available (see figure 5), increasing the OS channel in both models. From figure 24 we see that the OS/3b ratio should be a good discriminator up to $m_{\tilde{g}} \sim 450 \mathrm{GeV}$. For $m_{\tilde{g}} \gtrsim 450 \mathrm{GeV}$, higher luminosities are required in order to distinguish the models. But with high enough luminosities, we should be able to tell the models apart up to $m_{\tilde{g}} \sim 600 \mathrm{GeV}$. For even higher $m_{\tilde{g}}$ values, the signal features are too similar and the simple OS/3b ratio is no longer useful. 


\section{Comparison with CDF/CMS multijets $+E_{T}^{\text {miss }}$ channel}

Next we would like to compare our results for the multi-b-jets $+E_{T}^{\text {miss }}$ signature with a multijets $+E_{T}^{\text {miss }}$ analysis for SUSY searches proposed and used by CDF [99-101] and further developed in CMS [102]. We implement the following selection.

- $n($ jets $) \geq 3$ (we take jet $\left.p_{T}>50 \mathrm{GeV}\right)$,

- $E_{T}^{\text {miss }} \geq 150 \mathrm{GeV}$,

- $\Delta \phi\left(\vec{E}_{T}^{\mathrm{miss}}, \vec{H}_{T}\right)<1$,

- $0.5<R_{2} \equiv \sqrt{\Delta \phi^{2}\left(j 1, E_{T}^{\text {miss }}\right)+\left(\pi-\Delta \phi\left(j 2, E_{T}^{\text {miss }}\right)\right)^{2}}<4$,

- $1.5<R_{a 1} \equiv \sqrt{\left(\Delta \phi\left(j 2, E_{T}^{\text {miss }}\right)\right)^{2}+\left(\Delta \phi\left(j 3, E_{T}^{\text {miss }}\right)\right)^{2}}<4.25$,

- $\Delta \phi\left(j 2, E_{T}^{\mathrm{miss}}\right)>0.35$,

- $\Delta \phi\left(j_{i}, E_{T}^{\mathrm{miss}}\right)>0.3$,

- $|\eta(j 1)|<1.7$,

- Finally, we require the highest $E_{T}$ object in each signal event to be hadronic, rather than leptonic. ${ }^{10}$

The angular cuts are introduced in multijets $+E_{T}^{\text {miss }}$ analyses where no lepton information is explicitly used in order to guarantee the discrimination of QCD backgrounds which may have large $E_{T}^{\text {miss }}$ arising primarily due to jet mismeasurements. In such events, $E_{T}^{\text {miss }}$ typically aligns with the 2 nd hardest jet, as the hardest jet has a tendency to be mismeasured most and eventually becomes the 2 nd hardest jet.

The reach results for $\mathrm{CDF} / \mathrm{CMS}$ multijets $+E_{T}^{\text {miss }}$ cuts with no $n(b)$ requirement can be seen in figure 25 . The $5 \sigma$ reach for $0.2(1) \mathrm{fb}^{-1}$ of integrated luminosity is found to be $m_{\tilde{g}} \sim 370$ (430) $\mathrm{GeV}$.

In figure 26, we plot the $n(b)$ signal and background distributions after CDF/CMS cuts, which share similar characteristics with the distributions after $\mathrm{C} 1$ cuts. The dominance of signal starting with $n(b)=1$ illustrates the importance of considering $b$-jet tagging in the multijets $+E_{T}^{\text {miss }}$ analyses. The original CMS analysis made use of the $B$-triggers to significantly enhance the signal/BG ratio, while here we directly cut on $n(b)$. We show in table 5 the cross sections found after implementing CDF/CMS cuts with no requirement on $n(b)$ and with $n(b)=1,2,3$.

\footnotetext{
${ }^{10}$ This is an approximation for the leading track isolation step of the indirect lepton veto (ILV) technique included in the CDF/CMS selection. Normally in multijets $E_{T}^{\text {miss }}$ analyses where lepton information is not explicitly used, $W / Z / t \bar{t}+n$ jets backgrounds with leptonic $W, Z$ decays can be eliminated by vetoing the events whose leading track is isolated. We omit the second step of ILV featuring jet electromagnetic and charged fractions since it is designed to eliminate machine and cosmic backgrounds which we do not consider here.
} 


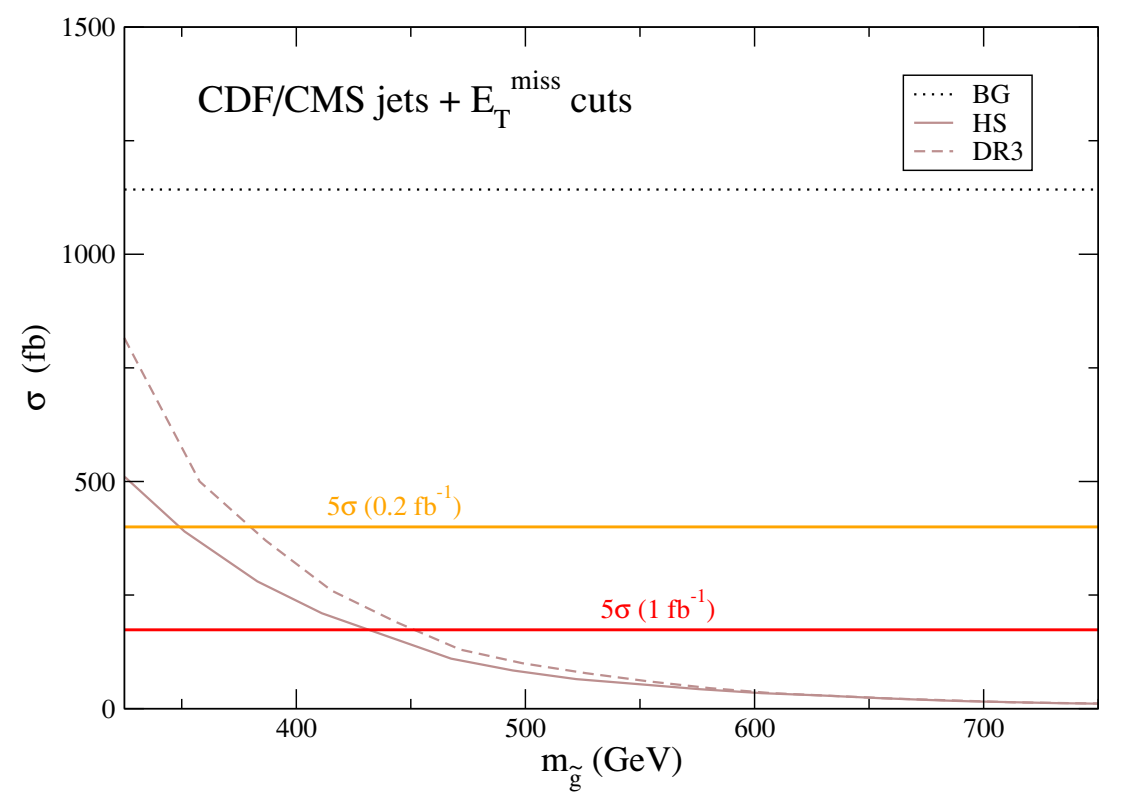

Figure 25. LHC reach for Yukawa-unified SUSY using CDF/CMS multijets $+E_{T}^{\text {miss }}$ cuts with no $n(b)$ requirement.

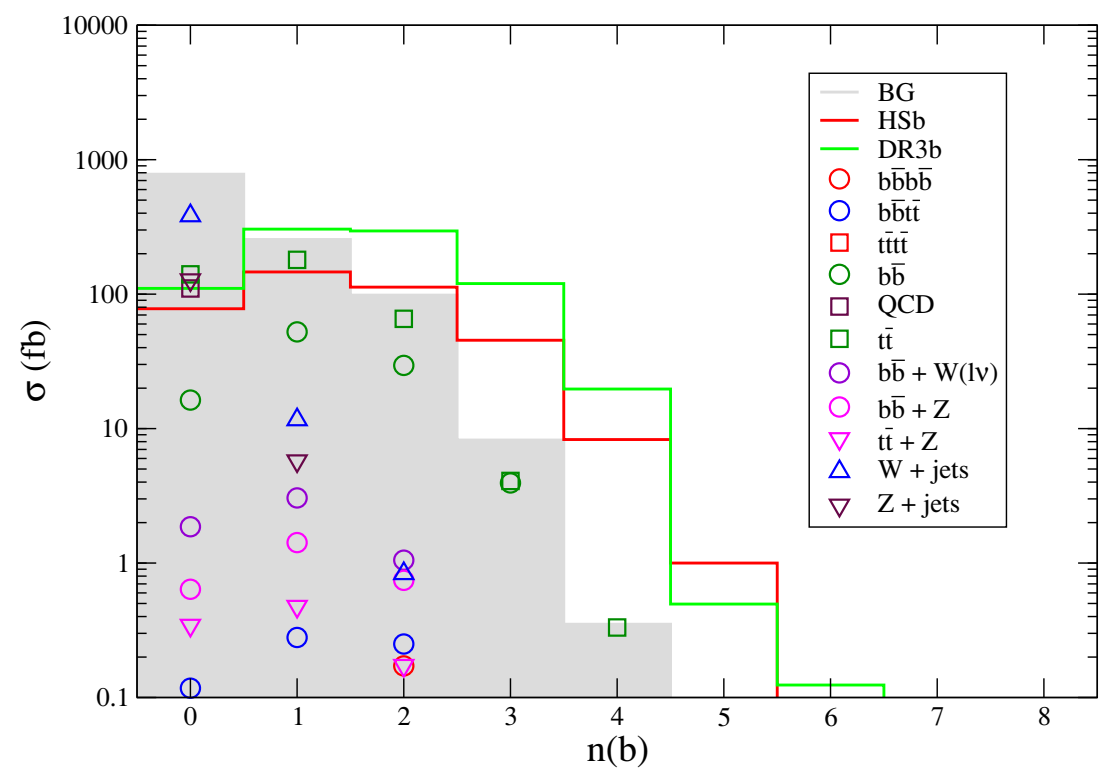

Figure 26. $b$-jet distribution after CDF/CMS multijets $+E_{T}^{\text {miss }}$ cuts at the LHC, with $\sqrt{s}=7 \mathrm{TeV}$. We show the signal levels for the HSb (red) and DR3b (green) points along with various SM backgrounds.

As before, we can do much better by requiring a high multiplicity of $b$-jets. In figure 27 , we adopt the same cuts as in figure 25 , but in addition require $n(b) \geq 3$. In this case, the BG drops by a factor of $\sim 150$, while the signal drops merely by a factor of $\sim 7$ for $m_{\tilde{g}} \sim 300 \mathrm{GeV}$. The LHC reach increases to $m_{\tilde{g}} \sim 400(500) \mathrm{GeV}$ for $0.2(1) \mathrm{fb}^{-1}$ of integrated luminosity. 


\begin{tabular}{|c|c|c|c|c|}
\hline \multicolumn{5}{|c|}{ Results after CDF/CMS multijets $+E_{T}^{\text {miss }}$-based selection } \\
\hline & $\sigma($ no $n(b)$ req. $)$ & $\sigma(n(b) \geq 1)$ & $\sigma(n(b) \geq 2)$ & $\sigma(n(b) \geq 3)$ \\
\hline HSb & $390 \mathrm{fb}$ & $313 \mathrm{fb}$ & $167 \mathrm{fb}$ & $55 \mathrm{fb}$ \\
\hline DR3b & $849 \mathrm{fb}$ & $739 \mathrm{fb}$ & $435 \mathrm{fb}$ & $140 \mathrm{fb}$ \\
\hline BG & $1132 \mathrm{fb}$ & $366 \mathrm{fb}$ & $101 \mathrm{fb}$ & $7 \mathrm{fb}$ \\
\hline
\end{tabular}

Table 5. Cross-sections for the channels with no $n(b)$ requirement, $n(b) \geq 1,2$ and 3 after the $\mathrm{CDF} / \mathrm{CMS}$ multijets $+E_{T}^{\text {miss }}$ cuts for the points HSb, DR3b and the background.

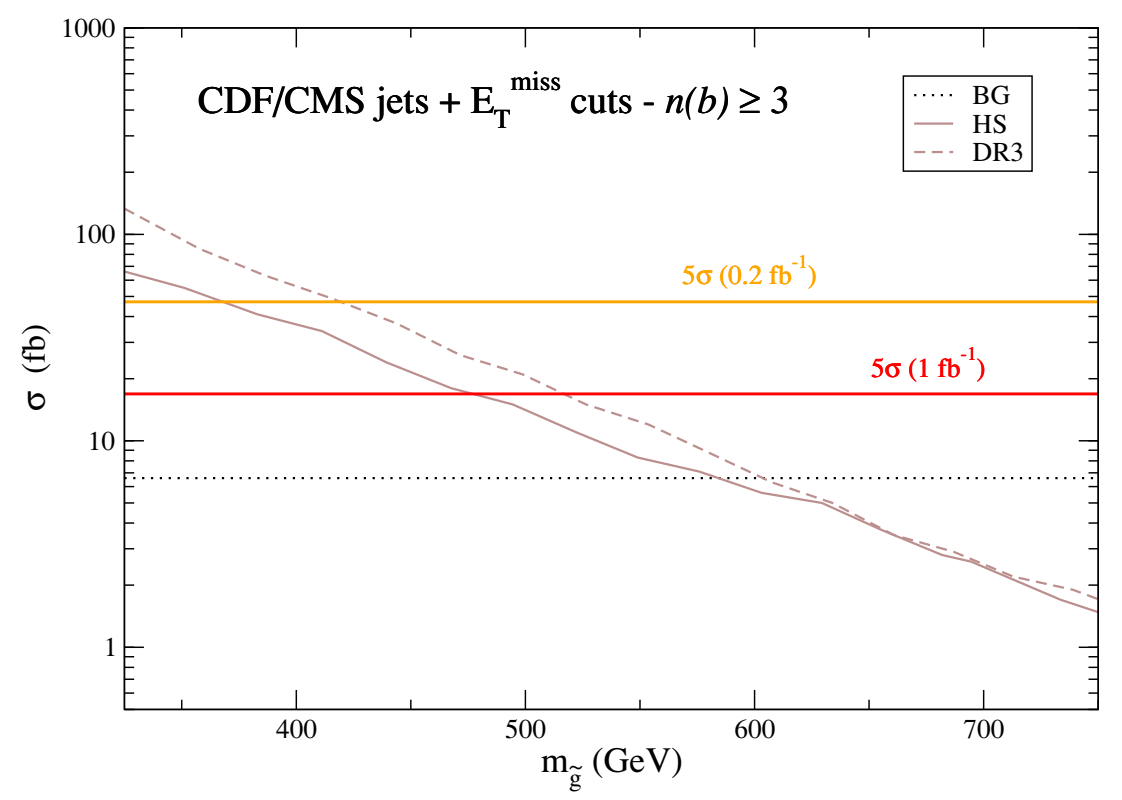

Figure 27. LHC reach for Yukawa-unified SUSY using CDF/CMS multijets $+E_{T}^{\text {miss }}$ cuts with $n_{b} \geq 3$.

\section{Conclusions}

In $t-b-\tau$ Yukawa-unified SUSY, we expect a characteristic spectrum of superpartners with first/second generation squarks and sleptons around $10 \mathrm{TeV}$, third generation sparticles, heavy Higgs bosons and $\mu$ around the few TeV level, and very light gauginos, with $m_{\tilde{g}} \sim$ $300-500 \mathrm{GeV}$ (although here we consider even higher values). Thus, at LHC, we expect to see gluino pair production at a high rate, followed by gluino decays to $b \bar{b} \widetilde{Z}_{i}$ or $t \bar{b} \widetilde{W}_{1}^{-}+$ c.c.. SUSY searches should therefore exploit the high multiplicity of $b$-jets expected in this scenario.

We investigated two model lines- the HS and DR3 cases. The HS case leads to large rates for OS/SF dileptons in the final state, while DR3 case does not. We computed numerous $2 \rightarrow 2,2 \rightarrow 3$ and $2 \rightarrow 4$ background processes. We found that with just $0.1-$ $0.2 \mathrm{fb}^{-1}$ of integrated luminosity, the LHC discovery reach with $\sqrt{s}=7 \mathrm{TeV}$ extends out to $m_{\tilde{g}} \sim 400 \mathrm{GeV}$, even without using $E_{T}^{\text {miss }}$ cuts. Crucial use is made of the high multiplicity of $b$-jets in the final state. In the case of the HS model, a corroborating signal appears in the $\mu^{+} \mu^{-}+$jets $+\geq 1 b$-jet channel.

The LHC reach at very low luminosity and without $E_{T}^{\text {miss }}$ is comparable to the Tevatron 


\begin{tabular}{|c|c|c|c|c|c|}
\hline & $\mathcal{L}\left(\mathrm{fb}^{-1}\right)$ & 0.05 & 0.1 & 0.2 & 1 \\
\hline \multirow{5}{*}{ C0 } & HS & $340 \mathrm{GeV}$ & $371 \mathrm{GeV}$ & $400 \mathrm{GeV}$ & $471 \mathrm{GeV}$ \\
& Channel & $n(b) \geq 3$ & $n(b) \geq 3$ & $n(b) \geq 3$ & $n(b) \geq 4$ \\
& DR3 & $340 \mathrm{GeV}$ & $363 \mathrm{GeV}$ & $394 \mathrm{GeV}$ & $469 \mathrm{GeV}$ \\
& Channel & $n(b) \geq 3$ & $n(b) \geq 3$ & $n(b) \geq 3$ & $n(b) \geq 3$ \\
\hline \multirow{5}{*}{ C1 } & HS & $436 \mathrm{GeV}$ & $480 \mathrm{GeV}$ & $526 \mathrm{GeV}$ & $630 \mathrm{GeV}$ \\
& Channel & $n(b) \geq 3$ & $n(b) \geq 3$ & $n(b) \geq 3$ & $n(b) \geq 3$ \\
& DR3 & $460 \mathrm{GeV}$ & $506 \mathrm{GeV}$ & $545 \mathrm{GeV}$ & $630 \mathrm{GeV}$ \\
& Channel & $n(b) \geq 3$ & $n(b) \geq 3$ & $n(b) \geq 3$ & $n(b) \geq 3$ \\
\hline \multirow{5}{*}{ CDF/CMS } & HS & - & $341 \mathrm{GeV}$ & $380 \mathrm{GeV}$ & $474 \mathrm{GeV}$ \\
& Channel & - & $n(b) \geq 1$ & $n(b) \geq 1$ & $n(b) \geq 3$ \\
& DR3 & $350 \mathrm{GeV}$ & $382 \mathrm{GeV}$ & $420 \mathrm{GeV}$ & $516 \mathrm{GeV}$ \\
& Channel & $n(b) \geq 2$ & $n(b) \geq 2$ & $n(b) \geq 2$ & $n(b) \geq 3$ \\
\hline
\end{tabular}

Table 6. Gluino $5 \sigma$ mass reach for the HS and DR3 model lines for different luminosites. We show the values for the early search $(\mathrm{C} 0$ cuts), the full reach $(\mathrm{C} 1$ cuts) and the $\mathrm{CDF} / \mathrm{CMS}$ multijets + $E_{T}^{\text {miss }}$ cuts (CDF/CMS). We also show the channel which optimizes the reach.

reach in the multi- $b+E_{T}^{\text {miss }}$ channel with $5-10 \mathrm{fb}^{-1}$ of data. This may lead to a tight competition for the discovery or exclusion of the simplest Yukawa-unified SUSY scenario!

Moving beyond about $0.2 \mathrm{fb}^{-1}$, we expect reliable $E_{T}^{\text {miss }}$ resolution and electron identification to become available. We find that the LHC reach, using $\sqrt{s}=7 \mathrm{TeV}$ and $1 \mathrm{fb}^{-1}$ of integrated luminosity, will move into the $m_{\tilde{g}} \sim 600-650 \mathrm{GeV}$ range for both the HS and DR3 model lines, if we require $E_{T}^{\text {miss }} \geq 100 \mathrm{GeV}$ along with $n(b) \geq 3$. This reach is presumably sufficient to rule out Yukawa unification in the DR3 case. In the HS case, somewhat larger values of $m_{\tilde{g}}$ can be allowed, although they seem very improbable. The LHC reach for $1 \mathrm{fb}^{-1}$ using the multi-jets $+E_{T}^{\text {miss }}$ signature- but making no requirement on $n(b)$ - turns out to be much lower. A summary of our various results is presented in a convenient form in table 6 .

At some point LHC energy will be increased to $\sim 10 \mathrm{TeV}$, and this will allow the reach to be extended past the values presented here. Thus, our main conclusion is that LHC stands an excellent chance to either discover Yukawa-unifed SUSY during year 1 of operation, or exclude almost all its model parameter space!

Last but not least we note that, if the scenario discussed here is realized, a trilepton signal from Drell-Yan $\widetilde{W}_{1} \widetilde{Z}_{2}$ production should appear when moving into the several $\mathrm{fb}^{-1}$ regime, giving direct access to the chargino/neutralino sector.

Note added. While finalizing this manuscript, we found an Atlas note [103] which also investigates using multiple $b$-jets to enhance the LHC reach for SUSY in the mSUGRA model. They examine integrated luminosity values of 0.1 and $1 \mathrm{fb}^{-1}$, but take $\sqrt{s}=14 \mathrm{TeV}$. Their overall results seem quite consistent with the results given here. 


\section{Acknowledgments}

We thank Harrison Prosper for sharing with us his p-value and significance code. This research was supported in part by the U.S. Department of Energy grant number DE-FG97ER41022, by the Fulbright Program and CAPES (Brazilian Federal Agency for PostGraduate Education). The work of SK is supported in part by the French ANR project ToolsDMColl, BLAN07-2-194882.

Open Access. This article is distributed under the terms of the Creative Commons Attribution Noncommercial License which permits any noncommercial use, distribution, and reproduction in any medium, provided the original author(s) and source are credited.

\section{References}

[1] R.N. Mohapatra, Unification and supersymmetry, hep-ph/9911272 [SPIRES].

[2] S. Raby, Desperately seeking supersymmetry [susy], Rept. Prog. Phys. 67 (2004) 755 [hep-ph/0401155] [SPIRES].

[3] H. Georgi, The state of the art-gauge theories, in Proceedings of the American Institute of Physics, edited by C. Carlson (1974).

[4] H. Fritzsch and P. Minkowski, Unified interactions of leptons and hadrons, Ann. Phys. 93 (1975) 193 [SPIRES].

[5] M. Gell-Mann, P. Ramond and R. Slansky, Color embeddings, charge assignments, and proton stability in unified gauge theories, Rev. Mod. Phys. 50 (1978) 721 [SPIRES].

[6] P. Minkowski, $\mu \rightarrow$ e $\gamma$ at a rate of one out of 1-billion muon decays?, Phys. Lett. B 67 (1977) 421 [SPIRES].

[7] M. Gell-Mann, P. Ramond and R. Slansky, Complex spinors and unified theories, in the proceedings of the Supergravity, September 27-29, Stony Brook, U.S.A. (1979), North-Holland, Amsterdam The Netherlands (1979).

[8] R.N. Mohapatra and G. Senjanović, Neutrino mass and spontaneous parity nonconservation, Phys. Rev. Lett. 44 (1980) 912 [SPIRES].

[9] B. Ananthanarayan, G. Lazarides and Q. Shafi, Top mass prediction from supersymmetric guts, Phys. Rev. D 44 (1991) 1613 [SPIRES].

[10] B. Ananthanarayan, G. Lazarides and Q. Shafi, Radiative electroweak breaking and sparticle spectroscopy with tan Beta approximately $=m_{t} / m_{b}$, Phys. Lett. B 300 (1993) 245 [SPIRES].

[11] G.W. Anderson, S. Raby, S. Dimopoulos and L.J. Hall, Precise predictions for $m_{t}, V_{c b}$ and $\tan \beta$, Phys. Rev. D 47 (1993) 3702 [hep-ph/9209250] [SPIRES].

[12] G. Anderson, S. Raby, S. Dimopoulos, L.J. Hall and G.D. Starkman, A systematic SO(10) operator analysis for fermion masses, Phys. Rev. D 49 (1994) 3660 [hep-ph/9308333] [SPIRES].

[13] V.D. Barger, M.S. Berger and P. Ohmann, The supersymmetric particle spectrum, Phys. Rev. D 49 (1994) 4908 [hep-ph/9311269] [SPIRES].

[14] M.S. Carena, M. Olechowski, S. Pokorski and C.E.M. Wagner, Electroweak symmetry breaking and bottom-top Yukawa unification, Nucl. Phys. B 426 (1994) 269 [hep-ph/9402253] [SPIRES]. 
[15] B. Ananthanarayan, Q. Shafi and X.M. Wang, Improved predictions for top quark, lightest supersymmetric particle and Higgs scalar masses, Phys. Rev. D 50 (1994) 5980 [hep-ph/9311225] [SPIRES].

[16] R. Rattazzi and U. Sarid, The unified minimal supersymmetric model with large Yukawa couplings, Phys. Rev. D 53 (1996) 1553 [hep-ph/9505428] [SPIRES].

[17] T. Blazek, M.S. Carena, S. Raby and C.E.M. Wagner, A global $\chi^{2}$ analysis of electroweak data in SO(10) SUSY GUTs, Phys. Rev. D 56 (1997) 6919 [hep-ph/9611217] [SPIRES].

[18] T. Blazek and S. Raby, Supersymmetric grand unified theories and global fits to low energy data, Phys. Lett. B 392 (1997) 371 [hep-ph/9611319] [SPIRES].

[19] T. Blazek and S. Raby, $b \rightarrow$ s $\gamma$ with large $\tan \beta$ in MSSM analysis constrained by a realistic SO(10) model, Phys. Rev. D 59 (1999) 095002 [hep-ph/9712257] [SPIRES].

[20] T. Blazek, S. Raby and K. Tobe, Neutrino oscillations in a predictive SUSY GUT, Phys. Rev. D 60 (1999) 113001 [hep-ph/9903340] [SPIRES].

[21] T. Blazek, S. Raby and K. Tobe, Neutrino oscillations in an $\mathrm{SO}(10)$ SUSY GUT with $\mathrm{U}(2) \times \mathrm{U}(1)^{n}$ family symmetry, Phys. Rev. D 62 (2000) 055001 [hep-ph/9912482] [SPIRES].

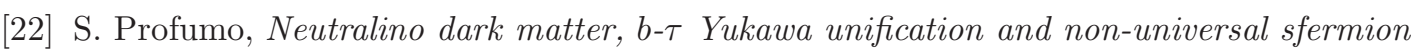
masses, Phys. Rev. D 68 (2003) 015006 [hep-ph/0304071] [SPIRES].

[23] C. Pallis, $b-\tau$ unification and sfermion mass non-universality, Nucl. Phys. B 678 (2004) 398 [hep-ph/0304047] [SPIRES].

[24] M.E. Gomez, G. Lazarides and C. Pallis, Supersymmetric cold dark matter with Yukawa unification, Phys. Rev. D 61 (2000) 123512 [hep-ph/9907261] [SPIRES].

[25] M.E. Gomez, G. Lazarides and C. Pallis, Yukawa quasi-unification, Nucl. Phys. B 638 (2002) 165 [hep-ph/0203131] [SPIRES].

[26] M.E. Gomez, G. Lazarides and C. Pallis, On Yukawa quasi-unification with $\mu<0$, Phys. Rev. D 67 (2003) 097701 [hep-ph/0301064] [SPIRES].

[27] U. Chattopadhyay, A. Corsetti and P. Nath, Supersymmetric dark matter and Yukawa unification, Phys. Rev. D 66 (2002) 035003 [hep-ph/0201001] [SPIRES].

[28] M.E. Gomez, T. Ibrahim, P. Nath and S. Skadhauge, WMAP dark matter constraints and Yukawa unification in SUGRA models with CP phases, Phys. Rev. D 72 (2005) 095008 [hep-ph/0506243] [SPIRES].

[29] H. Baer, M.A. Diaz, J. Ferrandis and X. Tata, Sparticle mass spectra from SO(10) grand unified models with Yukawa coupling unification, Phys. Rev. D 61 (2000) 111701 [hep-ph/9907211] [SPIRES].

[30] H. Baer et al., Yukawa unified supersymmetric SO(10) model: cosmology, rare decays and collider searches, Phys. Rev. D 63 (2001) 015007 [hep-ph/0005027] [SPIRES].

[31] H. Baer and J. Ferrandis, Supersymmetric SO(10) GUT models with Yukawa unification and a positive $\mu$ term, Phys. Rev. Lett. 87 (2001) 211803 [hep-ph/0106352] [SPIRES].

[32] T. Blazek, R. Dermisek and S. Raby, Predictions for Higgs and SUSY spectra from $\mathrm{SO}(10)$ Yukawa unification with $\mu>0$, Phys. Rev. Lett. 88 (2002) 111804 [hep-ph/0107097] [SPIRES].

[33] T. Blazek, R. Dermisek and S. Raby, Yukawa unification in $\mathrm{SO}(10)$, Phys. Rev. D 65 (2002) 115004 [hep-ph/0201081] [SPIRES]. 
[34] R. Dermisek, S. Raby, L. Roszkowski and R. Ruiz De Austri, Dark matter and $B_{s} \rightarrow \mu^{+} \mu^{-}$ with minimal $\mathrm{SO}(10)$ soft SUSY breaking, JHEP 04 (2003) 037 [hep-ph/0304101] [SPIRES].

[35] R. Dermisek, S. Raby, L. Roszkowski and R. Ruiz de Austri, Dark matter and $B_{s} \rightarrow \mu^{+} \mu^{-}$ with minimal $\mathrm{SO}(10)$ soft SUSY breaking. II, JHEP 09 (2005) 029 [hep-ph/0507233] [SPIRES].

[36] D. Auto et al., Yukawa coupling unification in supersymmetric models, JHEP 06 (2003) 023 [hep-ph/0302155] [SPIRES].

[37] G. Curio, Superpotentials for $M$-theory on a $G_{2}$ holonomy manifold and triality symmetry, JHEP 03 (2003) 024 [hep-th/0212211] [SPIRES].

[38] H. Baer, S. Kraml, S. Sekmen and H. Summy, Dark matter allowed scenarios for Yukawa-unified SO(10) SUSY GUTs, JHEP 03 (2008) 056 [arXiv:0801.1831] [SPIRES].

[39] W. Altmannshofer, D. Guadagnoli, S. Raby and D.M. Straub, SUSY GUTs with Yukawa unification: a go/no-go study using FCNC processes, Phys. Lett. B 668 (2008) 385 [arXiv: 0801.4363] [SPIRES].

[40] I. Gogoladze, R. Khalid and Q. Shafi, Yukawa unification and neutralino dark matter in $\mathrm{SU}(4)_{c} \times \mathrm{SU}(2)_{L} \times \mathrm{SU}(2)_{R}$, Phys. Rev. D $79(2009) 115004$ [arXiv: 0903. 5204] [SPIRES].

[41] D. Guadagnoli, S. Raby and D.M. Straub, Viable and testable SUSY GUTs with Yukawa unification: the case of split trilinears, JHEP 10 (2009) 059 [arXiv:0907.4709] [SPIRES].

[42] H. Baer, S. Kraml and S. Sekmen, Is 'just-so' Higgs splitting needed for t-b- $\tau$ Yukawa unified SUSY GUTs?, JHEP 09 (2009) 005 [arXiv: 0908.0134] [SPIRES].

[43] S.P. Martin and M.T. Vaughn, Two loop renormalization group equations for soft supersymmetry breaking couplings, Phys. Rev. D 50 (1994) 2282 [hep-ph/9311340] [SPIRES].

[44] R. Hempfling, Yukawa coupling unification with supersymmetric threshold corrections, Phys. Rev. D 49 (1994) 6168 [SPIRES].

[45] L.J. Hall, R. Rattazzi and U. Sarid, The Top quark mass in supersymmetric $\mathrm{SO}(10)$ unification, Phys. Rev. D 50 (1994) 7048 [hep-ph/9306309] [SPIRES].

[46] D.M. Pierce, J.A. Bagger, K.T. Matchev and R.-j. Zhang, Precision corrections in the minimal supersymmetric standard model, Nucl. Phys. B 491 (1997) 3 [hep-ph/9606211] [SPIRES].

[47] J.L. Feng, C.F. Kolda and N. Polonsky, Solving the supersymmetric flavor problem with radiatively generated mass hierarchies, Nucl. Phys. B 546 (1999) 3 [hep-ph/9810500] [SPIRES].

[48] J. Bagger, J.L. Feng and N. Polonsky, Naturally heavy scalars in supersymmetric grand unified theories, Nucl. Phys. B 563 (1999) 3 [hep-ph/9905292] [SPIRES].

[49] J.A. Bagger, J.L. Feng, N. Polonsky and R.-J. Zhang, Superheavy supersymmetry from scalar mass A-parameter fixed points, Phys. Lett. B 473 (2000) 264 [hep-ph/9911255] [SPIRES].

[50] H. Baer, P. Mercadante and X. Tata, Calculable sparticle masses with radiatively driven inverted mass hierarchy, Phys. Lett. B 475 (2000) 289 [hep-ph/9912494] [SPIRES].

[51] H. Baer et al., Aspects of supersymmetric models with a radiatively driven inverted mass hierarchy, Phys. Rev. D 64 (2001) 015002 [hep-ph/0102156] [SPIRES]. 
[52] H. Murayama, M. Olechowski and S. Pokorski, Viable t-b- $\tau$ Yukawa Unification in SUSY SO(10), Phys. Lett. B 371 (1996) 57 [hep-ph/9510327] [SPIRES].

[53] R.D. Peccei and H.R. Quinn, CP conservation in the presence of instantons, Phys. Rev. Lett. 38 (1977) 1440 [SPIRES].

[54] R.D. Peccei and H.R. Quinn, Constraints imposed by CP conservation in the presence of instantons, Phys. Rev. D 16 (1977) 1791 [SPIRES].

[55] S. Weinberg, A new light boson?, Phys. Rev. Lett. 40 (1978) 223 [SPIRES].

[56] F. Wilczek, Problem of strong $p$ and $t$ invariance in the presence of instantons, Phys. Rev. Lett. 40 (1978) 279 [SPIRES].

[57] J.E. Kim, Weak interaction singlet and strong CP invariance, Phys. Rev. Lett. 43 (1979) 103 [SPIRES].

[58] M.A. Shifman, A.I. Vainshtein and V.I. Zakharov, Can confinement ensure natural CP invariance of strong interactions?, Nucl. Phys. B 166 (1980) 493 [SPIRES].

[59] M. Dine, W. Fischler and M. Srednicki, A simple solution to the strong CP problem with a harmless axion, Phys. Lett. B 104 (1981) 199 [SPIRES].

[60] A.P. Zhitnitskii, On possible suppression of the axion hadron interactions. (In russian), Sov. J. Nucl. Phys. 31 (1980) 260 [Yad. Fiz. 31 (1980) 497] [SPIRES].

[61] J.E. Kim and G. Carosi, Axions and the strong CP problem, arXiv:0807.3125 [SPIRES].

[62] P. Sikivie, Axions 05, AIP Conf. Proc. 805 (2006) 23 [hep-ph/0509198] [SPIRES].

[63] M.S. Turner, Windows on the axion, Phys. Rept. 197 (1990) 67 [SPIRES].

[64] H.P. Nilles and S. Raby, Supersymmetry and the strong CP problem, Nucl. Phys. B 198 (1982) 102 [SPIRES].

[65] J.E. Kim and H.P. Nilles, The $\mu$ problem and the strong CP problem, Phys. Lett. B 138 (1984) 150 [SPIRES].

[66] J.E. Kim, A common scale for the invisible axion, local susy GUTs and saxino decay, Phys. Lett. B 136 (1984) 378 [SPIRES].

[67] G. Lazarides and Q. Shafi, Origin of matter in the inflationary cosmology, Phys. Lett. B 258 (1991) 305 [SPIRES].

[68] K. Kumekawa, T. Moroi and T. Yanagida, Flat potential for inflaton with a discrete $R$ invariance in supergravity, Prog. Theor. Phys. 92 (1994) 437 [hep-ph/9405337] [SPIRES]

[69] T. Asaka, K. Hamaguchi, M. Kawasaki and T. Yanagida, Leptogenesis in inflaton decay, Phys. Lett. B 464 (1999) 12 [hep-ph/9906366] [SPIRES].

[70] H. Murayama and T. Yanagida, Leptogenesis in supersymmetric standard model with righthanded neutrino, Phys. Lett. B 322 (1994) 349 [hep-ph/9310297] [SPIRES].

[71] M. Dine, L. Randall and S.D. Thomas, Baryogenesis from flat directions of the supersymmetric standard model, Nucl. Phys. B 458 (1996) 291 [hep-ph/9507453] [SPIRES].

[72] H. Baer, M. Haider, S. Kraml, S. Sekmen and H. Summy, Cosmological consequences of Yukawa-unified SUSY with mixed axion/axino cold and warm dark matter, JCAP 02 (2009) 002 [arXiv: 0812.2693] [SPIRES].

[73] H. Baer, S. Kraml, A. Lessa, S. Sekmen and H. Summy, Beyond the Higgs boson at the Tevatron: detecting gluinos from Yukawa-unified SUSY, arXiv:0910.2988 [SPIRES]. 
[74] H. Baer, S. Kraml, S. Sekmen and H. Summy, Prospects for Yukawa Unified SO(10) SUSY GUTs at the CERN LHC, JHEP 10 (2008) 079 [arXiv:0809.0710] [SPIRES].

[75] H. Goldberg, G. Perez and I. Sarcevic, Mini Z' burst from relic supernova neutrinos and late neutrino masses, JHEP 11 (2006) 023 [hep-ph/0505221] [SPIRES].

[76] D. Green, An LHC run plan: the first inverse femtobarn, hep-ph/0601038 [SPIRES].

[77] H. Baer, V. Barger and G. Shaughnessy, SUSY backgrounds to standard model calibration processes at the LHC, Phys. Rev. D 78 (2008) 095009 [arXiv:0806.3745] [SPIRES].

[78] J. Hubisz, J. Lykken, M. Pierini and M. Spiropulu, Missing energy look-alikes with $100 \mathrm{pb}^{-1}$ at the LHC, Phys. Rev. D 78 (2008) 075008 [arXiv:0805.2398] [SPIRES].

[79] M.L. Mangano, Standard model backgrounds to supersymmetry searches, Eur. Phys. J. C 59 (2009) 373 [arXiv:0809.1567] [SPIRES].

[80] H. Baer, H. Prosper and H. Summy, Early SUSY discovery at LHC without missing $E_{T}$ : the role of multi-leptons, Phys. Rev. D 77 (2008) 055017 [arXiv:0801.3799] [SPIRES].

[81] H. Baer, A. Lessa and H. Summy, Early SUSY discovery at LHC via sparticle cascade decays to same-sign and multimuon states, Phys. Lett. B 674 (2009) 49 [arXiv:0809.4719] [SPIRES].

[82] J. Edsjo, E. Lundstrom, S. Rydbeck and J. Sjolin, Early search for supersymmetric dark matter models at the LHC without missing energy, arXiv:0910.1106 [SPIRES].

[83] H. Baer, V. Barger, A. Lessa and X. Tata, Supersymmetry discovery potential of the LHC at $\sqrt{s}=10$ and $14 \mathrm{TeV}$ without and with missing $E_{T}, J H E P 09$ (2009) 063 [arXiv:0907.1922] [SPIRES].

[84] W. Beenakker, R. Hopker and M. Spira, PROSPINO: a program for the PROduction of Supersymmetric Particles In Next-to-leading Order QCD, hep-ph/9611232 [SPIRES].

[85] U. Chattopadhyay, A. Datta, A. Datta, A. Datta and D.P. Roy, LHC signature of the minimal SUGRA model with a large soft scalar mass, Phys. Lett. B 493 (2000) 127 [hep-ph/0008228] [SPIRES].

[86] P. Mercadante, K. Mizukohi and X. Tata, Using b-tagging to enhance the SUSY reach of the CERN Large Hadron Collider, Phys. Rev. D 72 (2005) 035009 [hep-ph/0506142] [spires.JPHRVA,D72,035009].

[87] S.P. Das et al., Focus Point SUSY at the LHC Revisited, Eur. J. Phys. C 54 (2008) 645 [arXiv: 0708.2048] [SPIRES].

[88] R. Kadala et al., Heavy-flavour tagging and the supersymmetry reach of the CERN Large Hadron Collider, Eur. J. Phys. C 56 (2008) 511 [arXiv:0803.0001] [SPIRES].

[89] H. Baer, C.-H. Chen, M. Drees, F. Paige and X. Tata, Collider phenomenology for supersymmetry with large tan $\beta$, Phys. Rev. Lett. 79 (1997) 986 [hep-ph/9704457] [SPIRES].

[90] H. Baer, C.-H. Chen, M. Drees, F. Paige and X. Tata, Supersymmetry reach of Tevatron upgrades: the large $\tan \beta$ case, Phys. Rev. D 58 (1998) 075008 [hep-ph/9802441] [SPIRES].

[91] M.L. Mangano, M. Moretti, F. Piccinini, R. Pittau and A.D. Polosa, ALPGEN, a generator for hard multiparton processes in hadronic collisions, JHEP 07 (2003) 001 [hep-ph/0206293] [SPIRES].

[92] T. Stelzer and W.F. Long, Automatic generation of tree level helicity amplitudes, Comput. Phys. Commun. 81 (1994) 357 [hep-ph/9401258] [SPIRES]. 
[93] T. Sjöstrand, S. Mrenna and P.Z. Skands, PYTHIA 6.4 physics and manual, JHEP 05 (2006) 026 [hep-ph/0603175] [SPIRES].

[94] F.E. Paige, S.D. Protopopescu, H. Baer and X. Tata, ISAJET 7.69: a Monte Carlo event generator for $p$, $\bar{p} p$ and $e^{+} e^{-}$reactions, hep-ph/0312045 [SPIRES].

[95] H. Baer, J. Ferrandis, S. Kraml and W. Porod, On the treatment of threshold effects in SUSY spectrum computations, Phys. Rev. D 73 (2006) 015010 [hep-ph/0511123] [SPIRES].

[96] H. Baer, C.-h. Chen, F. Paige and X. Tata, Signals for minimal supergravity at the CERN Large Hadron Collider II: multilepton channels, Phys. Rev. D 53 (1996) 6241 [hep-ph/9512383] [SPIRES].

[97] H. Baer, X. Tata and J. Woodside, $Z^{0}+$ jets + (missing) $p_{T}$ events as a signal for supersymmetry at the Tevatron collider, Phys. Rev. D 42 (1990) 1450 [SPIRES].

[98] H. Baer, C. Balázs, A. Belyaev, T. Krupovnickas and X. Tata, Updated reach of the CERN $L H C$ and constraints from relic density, $b \rightarrow s \gamma$ and $a(\mu)$ in the mSUGRA model, JHEP 06 (2003) 054 [hep-ph/0304303] [SPIRES].

[99] M. Spiropulu, A blind search for supersymmetry in $p \bar{p}$ collisions at $s^{1 / 2}=1.8 \mathrm{TeV}$ using the missing energy plus multijet channel, Ph.D. thesis, Harvard University, Cambridge U.S.A. (2000), UMI-99-88600.

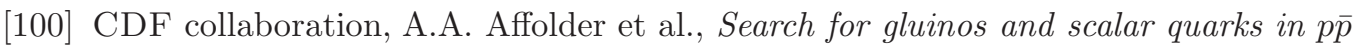
collisions at $\sqrt{s}=1.8 \mathrm{TeV}$ using the missing energy plus multijets signature, Phys. Rev. Lett. 88 (2002) 041801 [hep-ex/0106001] [SPIRES].

[101] CDF collaboration, A.A. Affolder et al., Search for gluinos and scalar quarks in $p \bar{p}$ collisions at $\sqrt{s}=1.8 \mathrm{TeV}$ using the missing energy plus multijets signature, Phys. Rev. Lett. 88 (2002) 041801 [hep-ex/0106001] [SPIRES].

[102] S. Sekmen, SUSY with heavy scalars at the LHC, Ph.D. thesis, Middle East Technical University (METU), Ankara, Turkey (2008), CMS TS-2009/025.

[103] ATLAS collaboration, Discovery potential for supersymmetry with b-jet final states with the Atlas detector, ATL-PHYS-PUB-2009-075 (2009). 